\title{
Is the astronomical forcing a reliable and unique pacemaker for climate? A conceptual model study
}

\author{
Bernard De Saedeleer • Michel Crucifix • \\ Sebastian Wieczorek
}

Received: 11 October 2011/Accepted: 22 January 2012/Published online: 20 April 2012

(C) The Author(s) 2012. This article is published with open access at Springerlink.com

\begin{abstract}
There is evidence that ice age cycles are paced by astronomical forcing, suggesting some kind of synchronisation phenomenon. Here, we identify the type of such synchronisation and explore systematically its uniqueness and robustness using a simple paleoclimate model akin to the van der Pol relaxation oscillator and dynamical system theory. As the insolation is quite a complex quasiperiodic signal involving different frequencies, the traditional concepts used to define synchronisation to periodic forcing are no longer applicable. Instead, we explore a different concept of generalised synchronisation in terms of (coexisting) synchronised solutions for the forced system, their basins of attraction and instabilities. We propose a clustering technique to compute the number of synchronised solutions, each of which corresponds to a different paleoclimate history. In this way, we uncover multistable synchronisation (reminiscent of phase- or frequency-locking to individual periodic components of astronomical forcing) at low forcing strength, and monostable or unique synchronisation at stronger forcing. In the multistable regime, different initial conditions may lead to different paleoclimate histories. To study their robustness, we analyse Lyapunov exponents that quantify the rate of
\end{abstract}

\footnotetext{
B. De Saedeleer $(\bowtie) \cdot$ M. Crucifix

Earth and Life Institute, Georges Lemaître Centre for Earth and Climate Research, Université catholique de Louvain, Louvain-la-Neuve, Belgium

e-mail: Bernard.DeSaedeleer@uclouvain.be

M. Crucifix

e-mail: Michel.Crucifix@uclouvain.be

S. Wieczorek

Mathematics Research Institute,

University of Exeter, Exeter, UK

e-mail: S.M.Wieczorek@exeter.ac.uk
}

convergence towards each synchronised solution (local stability), and basins of attraction that indicate critical levels of external perturbations (global stability). We find that even though synchronised solutions are stable on a long term, there exist short episodes of desynchronisation where nearby climate trajectories diverge temporarily (for about $50 \mathrm{kyr}$ ). As the attracting trajectory can sometimes lie close to the boundary of its basin of attraction, a small perturbation could quite easily make climate to jump between different histories, reducing the predictability. Our study brings new insight into paleoclimate dynamics and reveals a possibility for the climate system to wander throughout different climatic histories related to preferential synchronisation regimes on obliquity, precession or combinations of both, all over the history of the Pleistocene.

Keywords Climate models - Milankovitch - Oscillator . Generalised synchronisation - Lyapunov exponent . Multistability

\section{Introduction}

This article is a contribution to the field of paleoclimate dynamics theory, which has experienced many developments in terms of ice age models since many years notably by Le Treut and Ghil (1983), Saltzman and Maasch (1990), and many others, and remains an active research field. Paleoclimate modelling is a complex problem, hence an uncomfortable situation for a scientist. On the one hand, the paleoclimatic records are often difficult to interpret physically (these are only proxies), and an independent dating is not always easy to achieve; hence it leads to uncertainties both in magnitude and time. On the other 
hand, there is not a single well established model, the problem is non autonomous, the forcing is aperiodic, and stochastic effects are present.

Here, we focus on the slow variations of climate over the last few million years, which include the phenomenon of ice ages (Hays et al. 1976), that is, the repeated growth and decay of ice sheets in the Northern Hemisphere of a total mass as big as modern Antarctica's. When examining long-term climatic signals like the 5.3 Myr-long stack produced in Lisiecki and Raymo (2005), or the $800 \mathrm{kyr}$ long EPICA Dome C Ice Core from Luethi et al. (2008), plotted respectively in Fig. 1a, b for the last 500 kyr, one immediately identifies three clearly visible features of the climatic time series:

1. oscillations: the signal oscillates between higher and lower values of ice volume corresponding to the glacial and interglacial states,

2. asymmetry: in Fig. 1a typical transitions from a minimum to a maximum take much longer than transitions from a maximum to a minimum: deglaciations occur much more rapidly ( $\tau_{\text {fast }} \approx 10 \mathrm{kyr}$ ) than glaciations $\left(\tau_{\text {slow }} \approx 80 \mathrm{kyr}\right)$, giving a distinctive sawtooth structure in the glacial/interglacial (G/I) cycles, especially pronounced over the last $500 \mathrm{kyr}$,

3. 100-kyr dominant period: this has been identified by many authors since Broecker and van Donk (1970). Note that the G/I cycles are not periodic.

The asymmetry in the oscillations has been studied by many authors. In order to reproduce it, some authors use underlying physical principles to build phenomenological models that exhibit slow-fast dynamics reasonably mimicking the climatic proxies (Saltzman 2002). Others assume this asymmetry by explicitly defining 2 different parameters such as the time intervals $\tau_{u p}=\tau_{\text {slow }}$ and $\tau_{\text {down }}=\tau_{\text {fast }}$ (Ashkenazy 2006) or time constants $\left[\tau_{R}\right.$ and $\tau_{F}$ in Paillard (1998) and $T_{w}$ and $T_{c}$ in Imbrie and Imbrie (1980)]. Whatever the model, it has to ultimately exhibit asymmetric oscillations under the effect of the forcing, as it is aimed to mimic the oscillations between G/I states. Relaxation oscillators are therefore very straightforward natural candidates of ice age models (Crucifix 2012). In this article, we will consider a slightly modified van der Pol oscillator model to illustrate the new contributions of our synchronisation concepts.

In this article, we chose the best possible approach for dynamical modelling available so far, i.e. we assume that the observed G/I climate variability essentially emerges from externally forced low-dimensional deterministic dynamics possibly subject to stochastic disturbances. However, it is worth mentioning that alternative approaches have been developed to explain the G/I variability, e.g. the stochastic resonance-like phenomena ${ }^{1}$ (Benzi et al. 1982) in which high-dimensional (or at least approximately stochastic) dynamics plays a much more important role. A short discussion about this alternative approach is given in Crucifix (2012). It is very likely that future works should combine different approaches in some appropriate way.

In this paper, we concentrate on the influence of the astronomical forcing on Earth's climate. This forcing is induced by the slow variations in the spatial and seasonal distributions of incoming solar radiation (insolation) at the top of the atmosphere, associated with the slow variations of the Earth's astronomical elements: eccentricity $(e)$, true solar longitude of the perihelion measured with respect to the moving vernal equinox $(\varpi)$, and Earth obliquity $\left(\epsilon_{E}\right)$. These quantities are now accurately known over several tens of millions of years (Laskar et al. 2004), but analytical approximations of $e, e \sin \varpi$, and $\epsilon_{E}$ valid back to one million years have been known since Berger (1978). They take the form of d'Alembert series $\left(\sum A_{i} \sin \left[\omega_{i} t+\phi_{i}\right]\right)$.

The external forcing used throughout this article is the insolation at $65^{\circ} \mathrm{N}$ latitude on the day of the summer solstice. That specific insolation quantity is commonly related to the Milankovitch theory and can be thought of as a measure of how much ice may melt over summer. It can be written under the following compact form:

$F(t)=\frac{1}{a_{\epsilon_{1}}} \sum_{i=1}^{35}\left[s_{i} \sin \left(\omega_{i} t\right)+c_{i} \cos \left(\omega_{i} t\right)\right]$

where the value of the $3 \times 35$ parameters (including $\omega_{i}, s_{i}$, and $c_{i}$ ) are given in the Table 1 of "Appendix 1 ". The insolation has been scaled by $a_{\epsilon_{1}}$ in order to work dimensionless. The coefficients were extracted from Berger (1978) by performing a linear regression of the insolation on the $\omega_{i}$. The validity range of this approximation is $[-1,0] \mathrm{Myr}$, and its mean error (mean of the absolute value of the difference, compared to Laskar et al. (2004) is $6.7 \mathrm{~W} / \mathrm{m}^{2}$ with peaks at $27.5 \mathrm{~W} / \mathrm{m}^{2}$. Note that the mean value $\left(494.2447 \mathrm{~W} / \mathrm{m}^{2}\right)$ has been removed; the theoretical framework that allows to work with anomalies was justified by Saltzman and Maasch (1991). In short, this can be done as we are interested in oscillations, and not in the mean values themselves. The quasiperiodic ${ }^{2}$ nature of the insolation forcing $F(t)$ is illustrated in its spectral decomposition in Fig. 2. Precession is dominated by two harmonics around 19 and $23 \mathrm{kyr}(1 \mathrm{kyr}=1,000$ years $)$ and obliquity is dominated by a harmonic with a period of $41 \mathrm{kyr}$ but it bears periods as long as 1,200 kyr.

\footnotetext{
${ }^{1}$ Note that the stochastic resonance is also used in the modelling of the Dansgaard-Oeschger events (Ganopolski and Rahmstorf 2002; Braun et al. 2009).

${ }^{2}$ A quasiperiodic signal is the superposition of several periodic signals with incommensurate periods.
} 


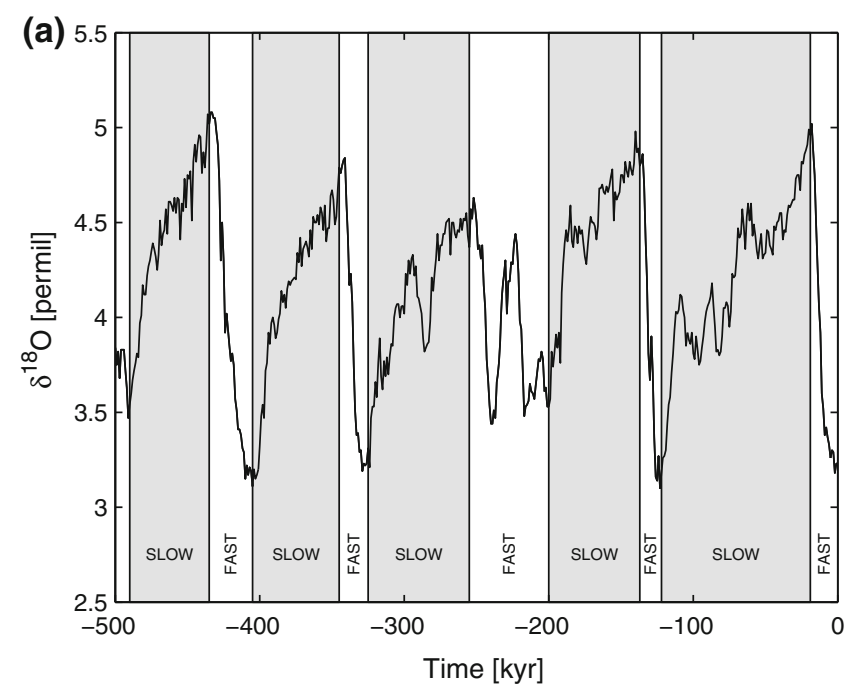

Fig. 1 The long-term climatic signals reveal a slow-fast dynamics (only the most recent $500 \mathrm{kyr}$ of the data are displayed here). The areas in grey (width $\tau_{\text {slow }}$ ) are wider than the areas in white (width $\left.\tau_{\text {fast }}\right)$ : while glaciation is a slow process of ice build-up, deglaciation occurs much more rapidly $\left(\tau_{\text {slow }}>\tau_{\text {fast }}\right)$. One also recognizes the last deglaciation which started some $20 \mathrm{kyr}$ ago, up to the present time

Many attempts have been made since the eighties in order to identify any relationship between the frequencies observed in the paleoclimatic records, reproduced by a given mathematical model, and those present in the insolation, mainly by performing spectral analysis. For example, (Le Treut and Ghil 1983) consider a nonlinear climatic oscillator based on physical climatic mechanisms, and found frequency locking for some specific runs of their model. They proposed to explain the ice age cycle in terms of a beat period (or combination tone) between the 19 and 23 kyr periods. Hyde and Peltier (1985) also propose a physical ice age model, and study several individual harmonic forcing periods (ibid., Fig. 23) and also astronomical forcing (Hyde and Peltier 1987) but reject the "combination tone" hypothesis. Paillard (1998) suggested two simple threshold models with multiple states in order to reproduce the nonlinearity between the $100 \mathrm{kyr}$ periodicity in the records and the insolation forcing. Gildor and Tziperman (2000) presented a sea ice climatic switch mechanism by which the insolation changes act as a pacemaker, setting the phase of the oscillations. Their box model is able to reproduce the asymmetric sawtooth structure, and results show phase-locking to the orbital variations through a nonlinear mechanism. Most of the time, however, the conclusions rely on a few particular realisations of the models, without providing a global analysis of the synchronisation phenomenon. Such an analysis is the subject of this study.

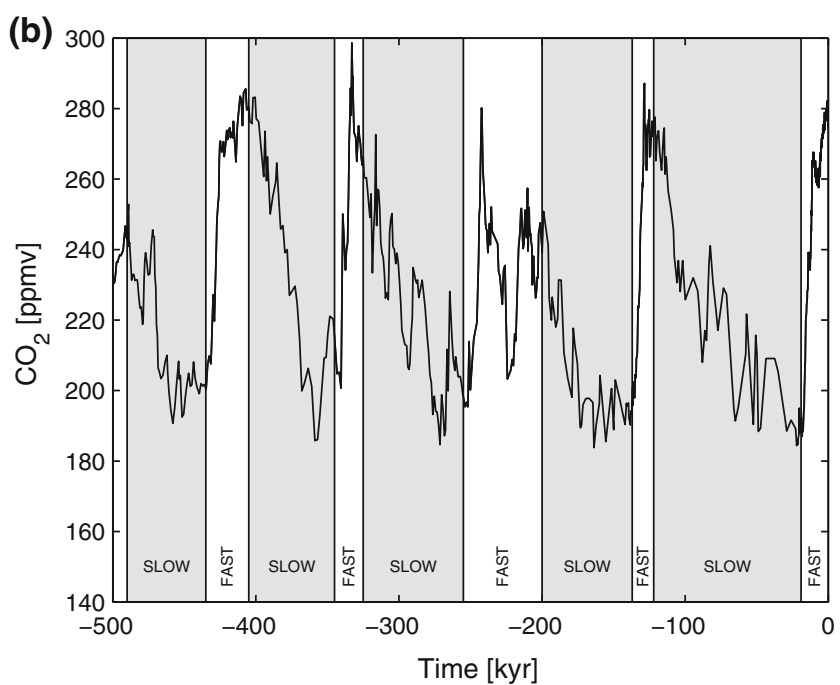

$(t=0)$. a The LR04 stack (Lisiecki and Raymo 2005) of 57 benthic $\delta^{18} O$ [\%o] records; the $\delta^{18} O$ is a proxy for the global volume of ice. High values of $\delta^{18} \mathrm{O}$ correspond to a colder climate (glacial state). b $\mathrm{CO}_{2}$ composite record [ppmv] (Luethi et al. 2008). High values of $\mathrm{CO}_{2}$ correspond to a warmer climate (interglacial state)

\section{Synchronisation}

There is ample evidence that the astronomical forcing influences the climate system. The phrase 'pacemaker of ice ages' was coined in a seminal paper (Hays et al. 1976) to express the idea that the timing of ice ages is controlled by the astronomical forcing, while the ice age cycle itself is shaped by internal system dynamics. The paradigm has prevailed since then and it is still supported by the most recent analyses of palaeoclimate records (Lisiecki and Raymo 2007; Huybers 2007). The notion of 'pacemaker' naturally evokes some sort of synchronisation. However, despite some attempts, the actual type of synchronisation has not been clearly identified or demonstrated to date. For example, (Ashkenazy 2006; Tziperman et al. 2006) speak of "nonlinear phase-locking" although they do not define suitable "phase variables" that can be used to demonstrate a fixed-in-time relationship between phases of the forcing and the oscillator response.

Synchronisation, as a universal nonlinear phenomenon, is a pervasive process in Nature, as it is associated with rhythmic processes. It is therefore not surprising to have synchronisation also in Paleoclimatic Sciences. Depending on the forcing type (periodic, chaotic, stochastic), one can distinguish many types of synchronisation including complete, lag, phase, frequency, identical, generalised (Rulkov et al. 1995), achronal and isochronous (Wu et al. 2006), and even noise synchronisation. For a review, the reader is referred to Balanov et al. (2009), Pikovsky et al. (2001), 


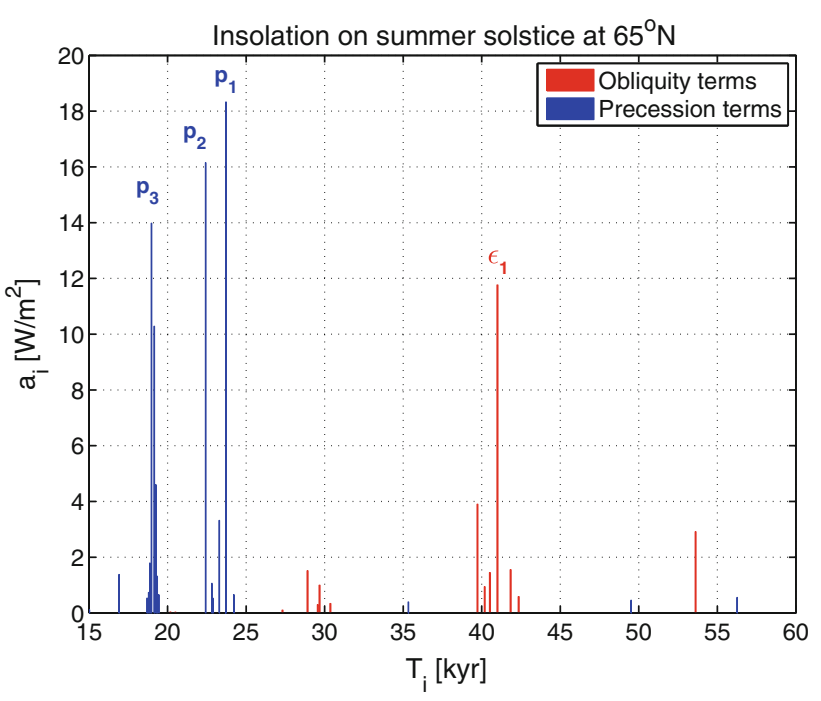

Fig. 2 Spectrum decomposition of the insolation at $65^{\circ} \mathrm{N}$ latitude on the day of the summer solstice $(F(t)$ given in Eq. 1). This is a graphical representation of Table 1 in "Appendix 1 ", with $a_{i}^{2}=s_{i}^{2}+c_{i}^{2}$ and $T_{i}=2 \pi / \omega_{i}$. The eight largest parameters $a_{i}$, which represent already $80 \%$ of the signal, are the major components of the insolation; they clearly come from the precession (19 and $23 \mathrm{kyr}$ ), and from the obliquity (41 kyr) associated series. This insolation is the forcing used to construct all figures subject to the quasiperiodic astronomical forcing. The main harmonic $\epsilon_{1}$ associated with obliquity has an angular frequency $\omega_{\epsilon_{1}}=0.1532 \mathrm{rad} / \mathrm{kyr}\left(T_{\epsilon_{1}}=41.0 \mathrm{kyr}\right)$ and an amplitude of $a_{\epsilon_{1}}=11.77 \mathrm{~W} / \mathrm{m}^{2}$. The three main harmonics associated with precession are denoted $p_{1}, p_{2}$ and $p_{3}$

among others. While some terminology is still debated, Brown and Kocarev (2000) proposed an unified definition of synchronisation for dynamical systems-there is synchronisation if there exists a relationship $h$ between the measured properties of the forcing, $g(\mathbf{u})$, and those of the oscillator, $g(\mathbf{v})$ :

$h(g(\mathbf{u}), g(\mathbf{v}))=0$,

that is fixed-in-time, meaning that $h$ is time independent. Because we are interested in synchronisation that is stable, for arbitrary initial conditions $\mathbf{u}(0)$ and $\mathbf{v}(0)$ that do not satisfy Eq. 2, we require that (Brown and Kocarev 2000):

$\lim _{t \rightarrow \infty} h(g(\mathbf{u}(t)), g(\mathbf{v}(t)))=0$.

For example, if $g(\mathbf{u})=\mathbf{u}, g(\mathbf{v})=\mathbf{v}, \mathbf{u}$ and $\mathbf{v}$ have the same dimension, and Eq. 2 can be written as $\mathbf{u}=\mathbf{v}$, we speak of identical synchronisation. More generally, if vectors $\mathbf{u}$ and $\mathbf{v}$ have different dimensions and Eq. 2 cannot be reduced to more than a functional relationship $\mathbf{u}=H(\mathbf{v})$, we speak of generalised synchronisation; see also Abarbanel et al. (1996), Rulkov et al. (1995) and Pikovsky et al. (2001). Note that the relationship (2) need not be unique. If there are two or more relationships (2) for the same parameter settings, we speak of multistable synchronisation
(Pikovsky et al. 2001, Ch.15.3.2). Then, which of the relationships the system settles to will depend on initial conditions.

In this paper, we use a simple van der Pol oscillator model to identify and illustrate for the first time the phenomenon of generalised synchronisation between ice age cycles and astronomical forcing. The dynamical systems approach outlined in the next section (1) allows for stability analysis of such synchronisation, (2) uncovers interesting effects related to the robustness of the synchronisation with respect to external perturbations, and (3) uncovers the phenomenon of multistable synchronisation that has been overlooked by previous studies. We show that, in contrast to claims in Tziperman et al. (2006), synchronisation needs not be unique.

The article is structured as follows. Section 2 introduces a slightly modified version of the van der Pol oscillator as a suitable model for studying synchronisation of ice ages to astronomical forcing. In Sect. 3, we analyse synchronisation to periodic forcing and quasiperiodic astronomical forcing in terms of largest Lyapunov exponents. Section 4 is dedicated to the study of multistable synchronisation in terms of attracting trajectories in the phase space of the forced system, and the associated basins of attraction. In Sect. 5, we investigate effects of the symmetry-breaking parameter $\beta$ for the van der Pol oscillator model. Section 6 is concerned with the robustness of the synchronisation and focuses on two aspects relating to predictability. Firstly, it shows that the local stability can be lost temporarily causing divergence of nearby climatic trajectories. Secondly, it demonstrates that in the multistable regime external perturbations (such as noise) may cause jumps between coexisting synchronised solutions when these solutions come close to their basin boundary. To be clear, all the treatment below is deterministic, except for Figs. 14 and 16.

This article requires some basics of Dynamical Systems theory (dynamical systems, nonlinear oscillations, limit cycles, bifurcations of vector fields, etc.), for which we refer the reader to Guckenheimer and Holmes (1983), Arnold (1983) and Strogatz (1994). We also refer to Saltzman (2002) for dynamical paleoclimatology, and to Savi (2005) for a review of many useful concepts such as attractors and Lyapunov exponents. For details about the van der Pol oscillator, we refer the reader mainly to van der Pol (1926), Strogatz (1994), Barnes and Grimshaw (1997), Hilborn (2000) and Balanov et al. (2009).

\section{Generic ice age model: a modified van der Pol relaxation oscillator}

The hypothesis at the basis of the work by Milankovitch (Milankovitch 1941) is that changes in the total amount of 
continental ice (say: $x$ ) are driven by summer insolation $F(t)$ already described in Eq. 1. One straightforward interpretation of this hypothesis is a simple differential equation $\dot{x}=-d \Psi(x) / d x-\gamma F(t)$, where $d \Psi(x) / d x$ is the derivative of a climatic potential and $\gamma$ is the forcing effciency. However, models of this form fail in practice to correctly capture the rapid deglaciation phenomenon. We therefore propose to model the paleoclimatic dynamical system with a dissipative self-sustained oscillator resembling the classical van der Pol oscillator ${ }^{3}$ :

$$
\begin{aligned}
\tau \dot{x} & =-[y+\beta-\gamma F(t)] \\
\tau \dot{y} & =-\alpha\left[\Phi^{\prime}(y)-x\right]
\end{aligned}
$$

where $\Phi^{\prime}(y)=y^{3} / 3-y$. The slow dynamics takes place on the slow manifold given by the function $\Phi^{\prime}(y)=x$. Note that this system is nonautonomous because the right-hand side depends explicitly on time. Throughout this article, we will also use a 41-kyr periodic forcing (main obliquity term) as a reference case; the corresponding forcing term in Eq. 4a will then be written under the form $[\gamma \sin (\omega t)]$ instead of $[\gamma F(t)]$.

The physical interpretation of the model $(4 \mathrm{a}, 4 \mathrm{~b})$ is as follows. Ice volume $x$ integrates the external forcing $F(t)$ over time but with a drift $y+\beta$. Assuming $\alpha \gg 1, y$ is the faster variable whose dynamics is controlled by a two-well potential $\Phi(y)$. For example, there are arguments that the dynamics of the Atlantic ocean circulation may be approximated by an equation similar to Eq. $4 \mathrm{~b}$ (Rahmstorf et al. 2005; Dijkstra et al. 2003). Further interpretation and discussion of the fast variable can be found in Saltzman et al. (1984), Tziperman and Gildor (2003), Paillard and Parrenin (2004), Tziperman et al. (2006) and Crucifix (2012). It is however not the goal of this paper to design the best suited paleoclimatic model; on the contrary, we propose a methodology for diagnosing synchronisation which could be applied to any paleoclimatic model, and which is illustrated herein using a simple conceptual paleoclimatic model sufficiently plausible. Also, we deliberately chose a deterministic approach in order to present the concepts, while it is clear that a more appropriate paleoclimatic model should also include stochastic components.

We introduce the parameter $\tau$ to have a control over the time scale of the oscillations (it is needed, as the parameters $\alpha$ and $\beta$ both affect the period of the unforced limit

\footnotetext{
3 The van der Pol oscillator, or slightly different versions of it [a similar one is the Poincaré oscillator (Glass and Sun 1994)], has been mathematically largely studied under many aspects, most of them being related to features used in the present study: fixed points and Arnol'd tongues, basins of attraction (Barnes and Grimshaw 1997), analytical expressions for the amplitude and period of the limit cycle (D'Acunto 2006), slow manifold equation (Ginoux and Rossetto 2006), bifurcation structure (Mettin et al. 1993), chaotic dynamics (Chen and Chen 2008; Parlitz and Lauterborn 1987), additive noise (Degli Esposti Boschi et al. 2002), etc.
}

cycle). The parameter $\beta$ controls the asymmetry of the glaciation/deglaciation sawtooth structure (a higher value of $\beta$ leads to an enhanced asymmetry), because it controls the position of the fixed-point on the slow manifold $\Phi^{\prime}(y)=y^{3} / 3-y=x$, and, consequently, the ratio of times spent by the system in the two branches ('glacial' and 'interglacial') of the slow manifold. The coupled system Eqs. $4 \mathrm{a}, 4 \mathrm{~b}$ has one stable equilibrium solution for $|\beta|>1$ and a stable periodic orbit for $|\beta|<1$. We use $T_{U L C}$ to denote the period of the stable periodic orbit and $\omega_{U L C}=$ $2 \pi / T_{U L C}$ to denote the corresponding angular frequency.

Relaxation oscillators have been proposed previously to study ice ages (Saltzman et al. 1984; Tziperman and Gildor 2003; Paillard and Parrenin 2004) although, to our knowledge, in a less general form than here. We adopted this form ${ }^{4}$ because it is very close to the well-studied van der Pol oscillator, and a reasonable qualitative agreement (timing of glacial inceptions and terminations, and their amplitude) with ice volume proxies was easily found for well chosen values of $\alpha, \beta, \gamma$ and $\tau$ (Fig. 3). We note, though, that small changes in parameters or additive fluctuations may easily shift the timings of ice-age terminations or glacial inceptions for reasons that will be clarified later in the paper. When driven, the van der Pol oscillator can lead to synchronisation (Balanov et al. 2009), but also to deterministic chaos (Ruihong et al. 2008), depending of the level of the driving force.

The definition of synchronisation can be applied to our model Eqs. 4a, 4b as follows. The astronomical forcing $F(t)$ corresponds to $\mathbf{u}(t)$, and the state vector whose two components are the slowly-varying ice volume $x$ and the faster variable $y$ corresponds to $\mathbf{v}(t)$. For nonperiodic forcing, relationship (2) can be very complicated (nonfunctional or even fractal-like) and hence difficult to detect. Therefore, other methods of detecting (2) had to be developed. As suggested by the auxiliary system approach (Abarbanel et al. 1996), relationships (2) and (3) are implied by an (invariant) attracting trajectory in the $(x, y, t)$ phase space of the nonautonomous forced system (4a, 4b) (Wieczorek 2011). In the remainder of the paper, such an attracting trajectory is denoted with $A T$ and referred to as an attracting climatic trajectory or synchronised solution. All other solutions to Eqs. $4 \mathrm{a}, 4 \mathrm{~b}$ will be referred to as climatic trajectories.

Previous approaches to nonlinear dynamics of quasiperiodically forced oscillators focused on discrete-time mappings and two-frequency forcing (Glendinning and Wiersig 1999; Osinga et al. 2000; Belogortsev 1992; Broer and Simó

\footnotetext{
${ }^{4}$ Note that the van der Pol oscillator model is also used as a reference e.g. in Saltzman (2002, p. 101) for a coupled ocean/sea-ice model, and also in Rial and Yang (2007) and Rial and Saha (2011) to simulate high resolution ice core data with a van der Pol-based coupled ocean/sea-ice model.
} 
1998). They uncovered interesting dynamics including Arnol'd or mode-locked tongues consisting of 'interlocking' bubbles and open regions of multistability, nonsmooth bifurcations, and strange nonchaotic attractors (Grebogi
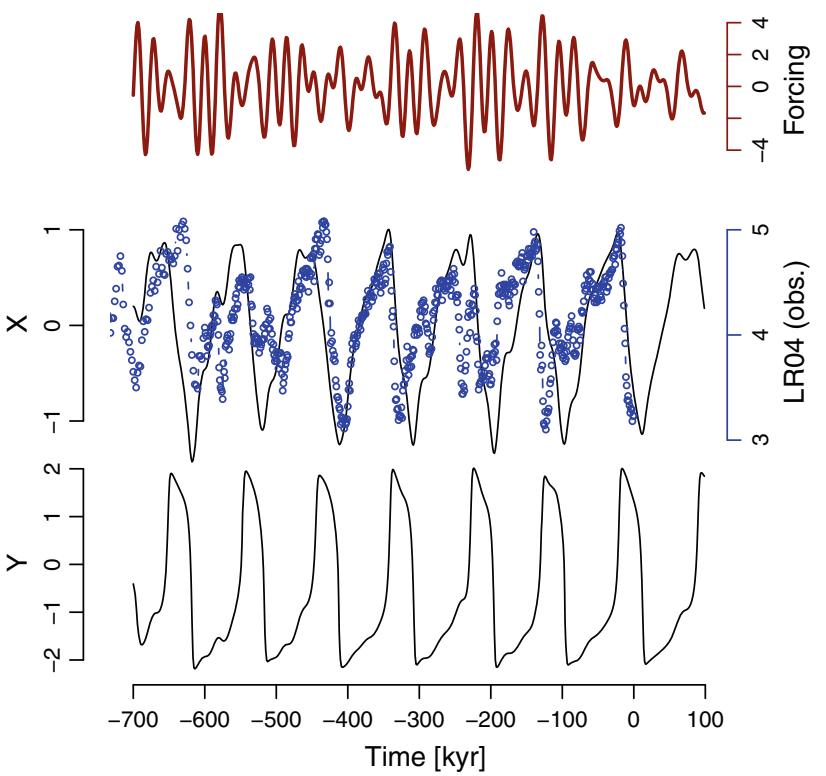

Fig. 3 Top: the insolation forcing $F(t)$. Bottom: the $x$ and $y$ climatic trajectories obtained using system Eqs. 4a, 4b with $\alpha=11.11$, $\beta=0.25, \gamma=0.75$ and $\tau=35.09$. With these parameters, $\omega_{\epsilon 1}=$ $2.5 \omega_{U L C}$, where $\omega_{\epsilon 1}$ is the angular frequency associated with the dominant harmonic of obliquity and $\omega_{U L C}$ is the angular frequency associated with the unforced system's periodic orbit. Blue dots correspond to the Lisiecki and Raymo stack (LR04) described in Fig. 1a. Time $t=0$ corresponds conventionally to the year 1950 . The model is in reasonable qualitative agreement with the ice volume proxy
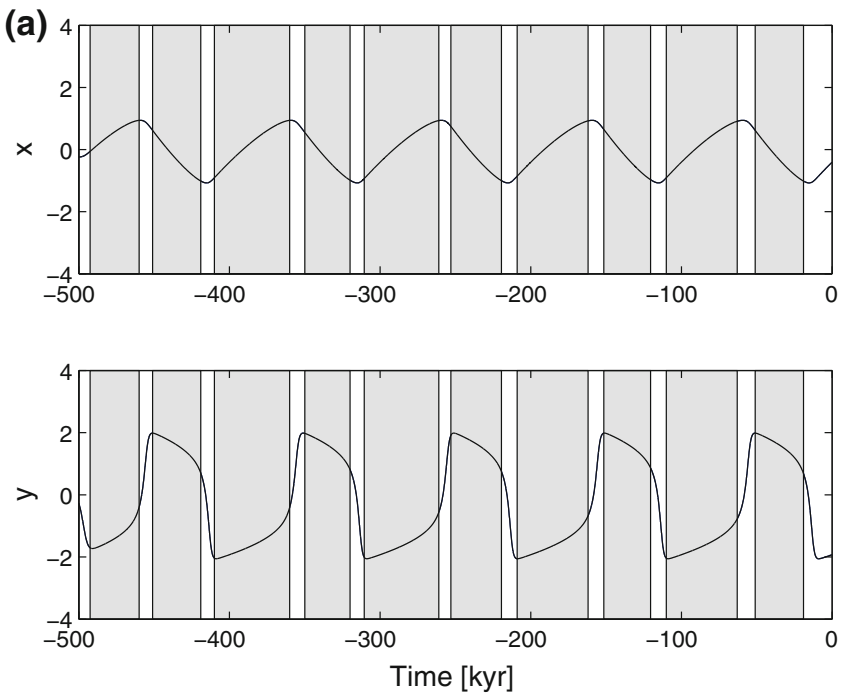

Fig. 4 Dynamics of the unforced ice ages model Eqs. 4a, 4b with $\alpha=11.11, \beta=0.25, \gamma=0$ and $\tau=35.09$. The slow-fast variable is $y$, while $x$ is always slow. Example of a typical climatic trajectory with initial conditions $\left(x_{-500}, y_{-500}\right)=(-0.24,-0.27)$. a Time series : $x$ (the ice volume) is the slow variable, while $y$ exhibits slow-fast dynamics. The same colour convention white/grey as in Fig. 1 has been used in et al. 1984; Feudel et al. 1995). Here, we consider quasiperiodic forcing with 35 frequency components and focus on the regions of mode locking. Our approach is based on instabilities of attracting trajectories in the $(x, y, t)$ phase space of the continuous-time forced system because they relate directly to the concept of generalised synchronisation. We can provide a systematic study of generalised synchronisation to astronomical forcing by demonstrating existence of such trajectories and exploring their local and global stability properties. More specifically, we perform three types of calculations. Firstly, a clustering detection technique uncovers parameter regions with monostable (unique) and multistable (non-unique) synchronisation. Secondly, the largest Lyapunov exponent along AT quantifies its long- and short-term local (linear) stability. Thirdly, a basin of attraction of $A T$ quantifies its global (nonlinear) stability. Finally, we remark that in the theory of nonautonomous dynamical systems, attracting trajectories in the $(x, y, t)$ phase space are related to a modern and more general concept of a pullback attractor (Kloeden 2000; Langa et al. 2002; Kosmidis and Pakdaman 2003; Wiggins 2003).

\section{Synchronisation of the paleoclimatic system to the insolation forcing}

\subsection{Illustration of the synchronisation phenomenon}

A typical climatic trajectory for $\gamma=0$ is shown in Fig. 4, from two different points of view: the time series and the

(b)

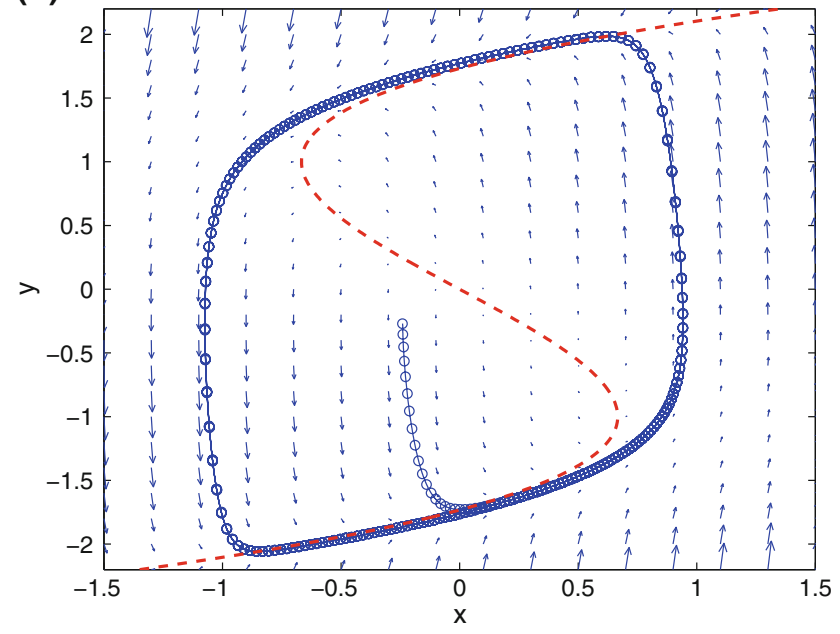

order to highlight the slow and fast episodes. b Phase space portrait in the two-dimensional $(x, y)$ phase space of the autonomous system: dynamical flow, limit cycle and residence plot (circles are spaced at every 0.5 kyr). More time is spent along the slow manifold (red dashed curve, corresponding to the function $\left.\Phi^{\prime}(y)=y^{3} / 3-y=x\right)$. The trajectory converges to the limit cycle (thin curve) with slow-fast dynamics 
phase space portrait. In the time series, we recognize the slow variable $x$ (the ice volume), while $y$ exhibits slow-fast dynamics. This climatic trajectory is also shown in the twodimensional $(x, y)$ phase space of the autonomous system where arrows indicate direction of the flow. The trajectory converges to the limit cycle with slow-fast dynamics (the speed along the trajectory can be visually assessed by the circles of the residence plot). Let us now consider a set of 70 random initial conditions in the $(x, y)$-plane at time $t_{0}=0$, and study the resulting climatic trajectories in the three-dimensional phase space $(x, y, t)$ of the nonautonomous system (Fig. 5a) for time $t>t_{0}$. One clearly sees that all trajectories converge to a cylinder-the attracting set in the $(x, y, t)$ space.

However, if we consider now an external forcing $(\gamma>0)$ then synchronisation onto this forcing may occur under certain conditions (Ashkenazy 2006; Tziperman et al. 2006). According to our definition (2-3), synchronisation is represented by an attracting climatic trajectory in the $(x, y, t)$ phase space.

Consider first the case of a purely periodic forcing with a period of $T_{F}=41 \mathrm{kyr}$ (main obliquity term) and strength $\gamma=3.33$. The 70 initial conditions give rise to climatic trajectories that, after a sufficiently long integration time, converge to two attracting trajectories (see Fig. 5c). Both attracting trajectories are periodic with period of $T_{R}=2 T_{F}=82 \mathrm{kyr}$, and time-shifted versions of each other. This phenomenon is described in the literature as 2:1 phase-locking or frequency-locking. Generally speaking a $n: m$ frequency-locking is defined as a fixed-in-time relation between the frequencies of the forcing $\left(\omega_{F}\right)$ and the oscillator response $\left(\omega_{R}\right)$ of the form $n \omega_{R}=m \omega_{F}$, where $m$ and $n$ are integers (Pikovsky et al. 2001, p. 52).

Then consider the case of the quasiperiodic insolation forcing described in Eq. 1 with $\gamma=0.75$ and $\tau=43.86$. Figure $5 \mathrm{e}$ shows that the 70 climatic trajectories now converge onto three attracting trajectories, which reveals that synchronisation can be multistable (Pikovsky et al. 2001, p. 348), (Balanov et al. 2009, p. 94). This phenomenon is described in the literature as mode-locking (Glass and Mackey 1988; Svensson and Coombes 2009). Note that because of the quasiperiodicity of the insolation forcing, these attracting trajectories are no longer periodic nor time-shifted versions of each other. The number of attracting trajectories depends on many factors including the dynamics of the unforced system, the nature of the forcing $F(t)$, and the amplitude $\gamma$ of the forcing. We will study this in more details in Sect. 4.

\subsection{Detection of synchronisation by the way} of the largest Lyapunov exponent (LLE or $\lambda_{\max }$ )

Local or linear stability of an attracting climatic trajectory can be quantified with the largest Lyapunov exponent
(LLE) denoted here as $\lambda_{\max }$ (Benettin et al. 1980). The quantity $\lambda_{\max }$ is a measure of the (average) exponential rate of divergence $\left(\lambda_{\max }>0\right)$ or convergence $\left(\lambda_{\max }<0\right)$ of nearby climatic trajectories. Therefore, a negative value of $\lambda_{\max }$ indicates a locally attracting climatic trajectory or generalised synchronisation (Pikovsky et al. 2001; Wieczorek 2009). A transition from $\lambda_{\max }<0$ to $\lambda_{\max }=0$ indicates a bifurcation where the attracting climatic trajectory disappears and generalised synchronisation is lost. Null and positive values of $\lambda_{\max }$ indicate lack of synchrony (positive $\lambda_{\max }$ indicates chaos but this regime is not encountered here). In the case of periodic forcing, computations of $\lambda_{\max }$ can be easily validated with more precise and reliable numerical bifurcation continuation techniques (see §' 41-kyr periodic forcing' below).

\subsection{Long-term $\lambda_{\max }$ and short-term $\lambda_{\max }^{H}$ LLE's}

The largest Lyapunov exponent $\lambda_{\max }$ is mathematically defined $^{5}$ as (Ott 2002) :

$\lambda_{\max }=\lim _{|\delta \mathbf{Z}(0)| \rightarrow 0} \lim _{t \rightarrow \infty} \frac{1}{t} \ln \frac{|\delta \mathbf{Z}(t)|}{|\delta \mathbf{Z}(0)|}$

where $\delta \mathbf{Z}=[\delta x, \delta y]$ are vanishing perturbations about $x$ and $y$, respectively, governed by the linearisation of system Eqs. $4 \mathrm{a}, 4 \mathrm{~b}$. Whereas this classical $\lambda_{\max }$ is defined in long term limit $(t \rightarrow \infty)$, one can also define (Abarbanel et al. 1991) a short-term version, $\lambda_{\max }^{H}$, by considering a finite time interval $H(H=50 \mathrm{kyr}$ will be considered in this article):

$\lambda_{\text {max }}^{H}=\lim _{|\delta \mathbf{Z}(0)| \rightarrow 0} \frac{1}{H} \ln \frac{|\delta \mathbf{Z}(H)|}{|\delta \mathbf{Z}(0)|}$

While $\lambda_{\max }$ gives the average or long-term stability information, $\lambda_{\max }^{H}$ can tell us about the behaviour of nearby trajectories within a short time interval $H$. For example, $\lambda_{\max }<0$ does not necessarily imply $\lambda_{\max }^{H}<0$ for some suitably chosen $H$. The definition (6) will be useful in studying the robustness of generalised synchronisation in Sect. 6. Technical details for computing $\lambda_{\max }$ are given in "Appendix 2".

\subsection{Influence of the parameters $\gamma$ and $T_{U L C}$}

The two particular types of synchronisation illustrated in Fig. 5c, e have been obtained for a fixed value of the amplitude $\gamma$ of the external forcing and of the natural period

\footnotetext{
5 Even if differential versions of the LLE have sometimes been developed mainly for computational efficiency purposes, we however preferred within this article to stick on the original definition of the LLE, because it is more standard and there is no insistent need for lowering computation time in the present framework, as the number of degrees of freedom of the system is reduced.
} 
(a)

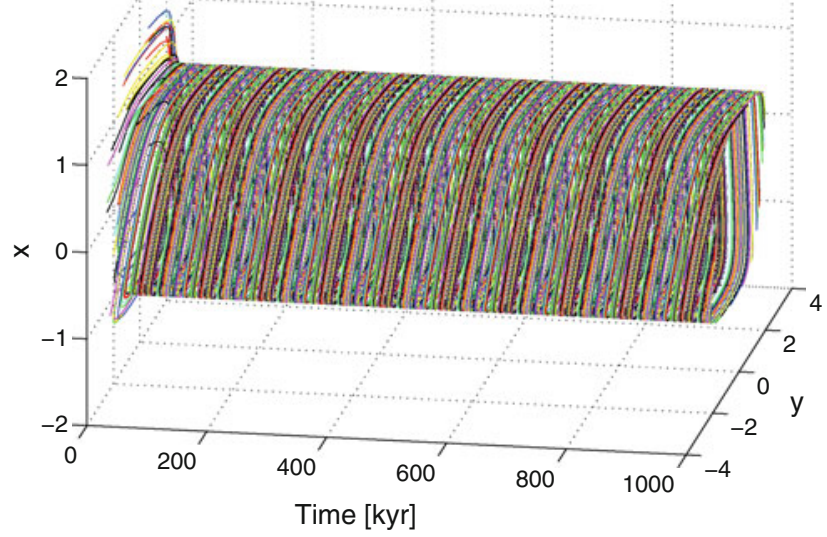

(c)

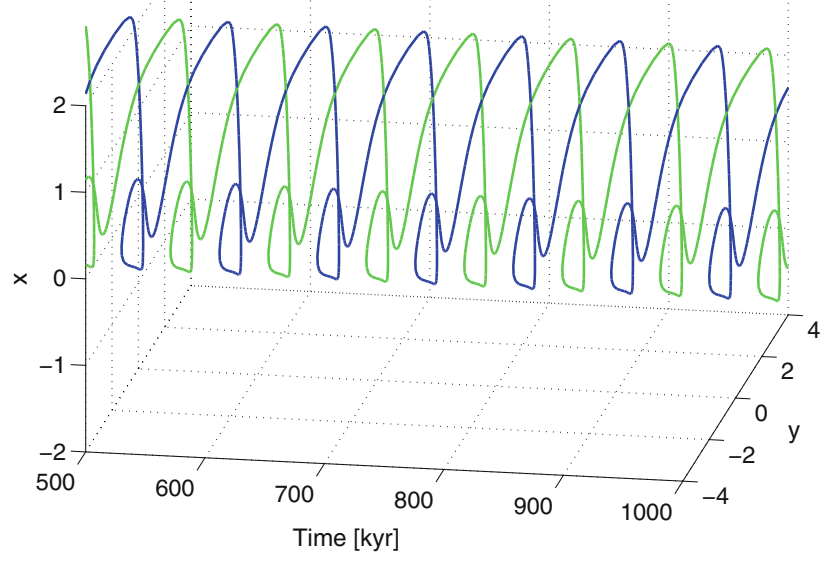

(e)

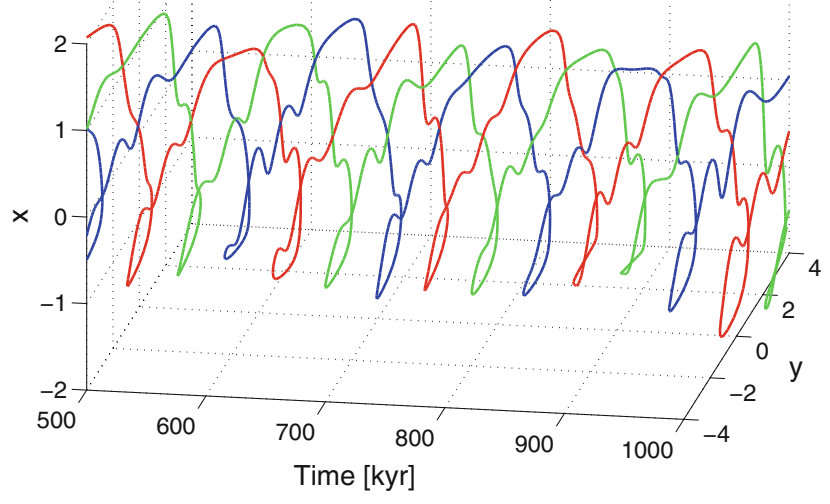

(b)

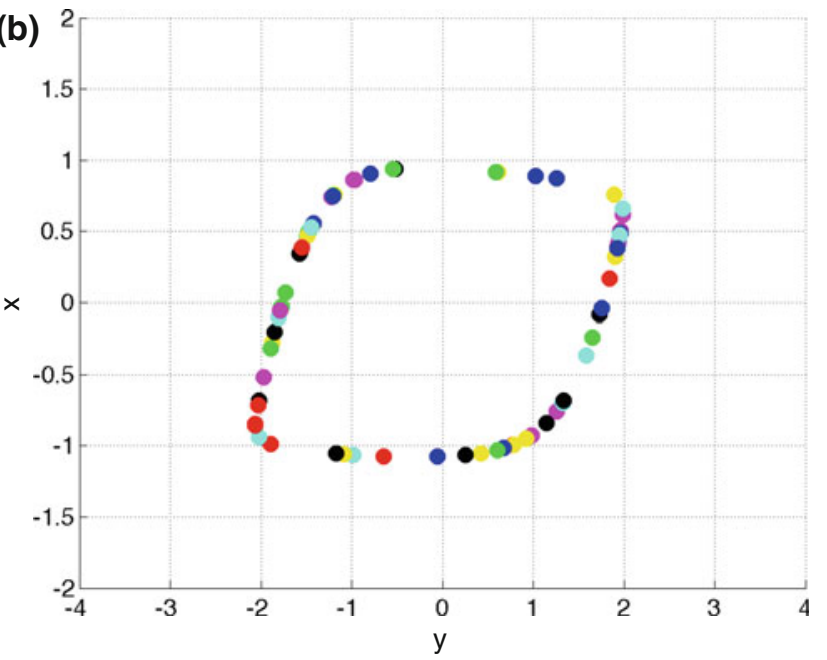

(d)

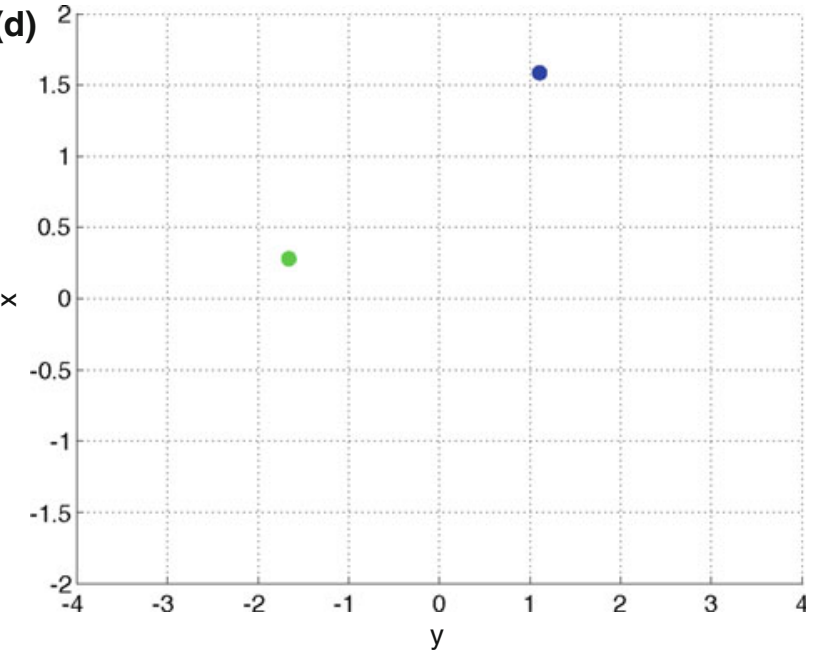

(f)

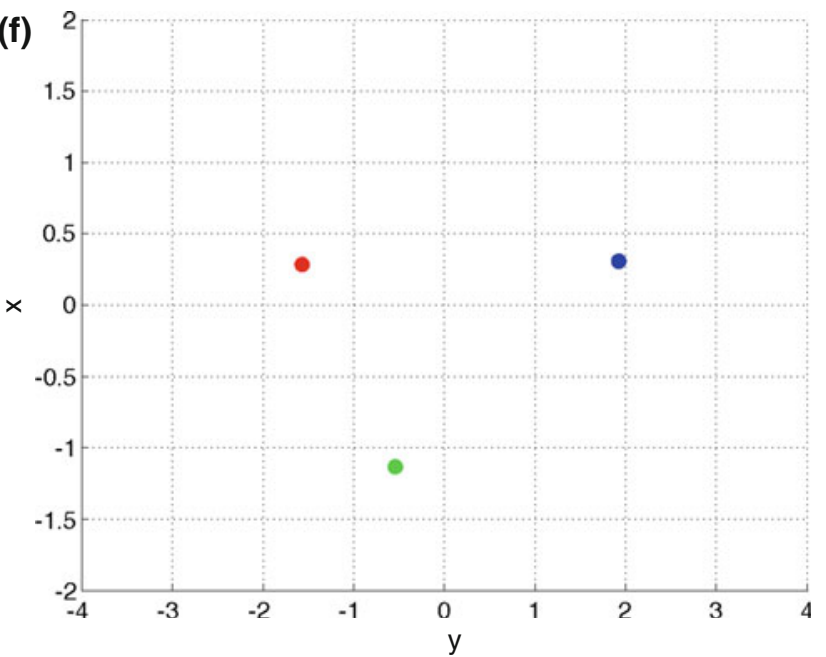


4 Fig. 5 Illustration of three different paleoclimate dynamical regimes: (top) without any forcing $\left(\gamma=0, \tau=35.09 \rightarrow T_{U L C} \approx 100 \mathrm{kyr}\right)$, (middle) 41-kyr periodic forcing $\left(\gamma=3.33, \tau=35.09 \rightarrow T_{U L C} \approx 100\right.$ kyr), (bottom) generalised multistable synchronisation on the quasiperiodic insolation forcing $\left(\gamma=0.75, \tau=43.86 \rightarrow T_{U L C} \approx 125 \mathrm{kyr}\right)$. The 41-kyr periodic forcing case $\mathbf{c}, \mathbf{d}$ is represented by the symbol ' + ' in Fig. 6a, c, and the quasiperiodic insolation forcing case $\mathbf{e}, \mathbf{f}$ by the symbol ' $x$ ' in Figs. 6b, d. The ice age model used is Eqs. 4a, 4b with $\alpha=11.11$ and $\beta=0.25$. A set of 70 random initial conditions at $t_{0}=0$ is used. Left: climatic trajectories in the $(x, y, t)$ space-time space. Right: section of these trajectories at $t=550 \mathrm{kyr}$, which reveals clearly the potential formation of the attracting trajectories and allows an easier counting of these. a All trajectories converge to a cylinder-the attracting set in the $(x, y, t)$ space. b No clear dense cluster of climatic trajectories is identified: no attracting trajectory exists. c The trajectories converge to two attracting trajectories of period 82 kyr: there is a frequency-locking 2:1. d Two clusters are identified, corresponding to the two attracting trajectories born from frequency-locking 2:1. e The trajectories converge now to three attracting trajectories for a long time, revealing a multistable synchronisation. f Three clusters of trajectories are identified, corresponding to the three attracting trajectories born from multistable synchronisation

of the unforced paleoclimatic system $T_{U L C}$. Now, we are equipped to achieve a much broader view of the dynamics by performing a parametric study on these two parameters. The quantity plotted in Fig. 6a, b is the largest Lyapunov exponent at $3 \mathrm{Myr}$, far from the transient behaviour so that $\lambda_{\max }^{H=3 \mathrm{Myr}}$ may already been considered as a good approximation of $\lambda_{\max }$.

\subsection{1-kyr periodic forcing (main obliquity term)}

Figure 6a corresponds to the case of the 41-kyr periodic forcing $\left(T_{F}=41 \mathrm{kyr}\right)$. The synchronisation region $\left(\lambda_{\max }<0\right)$ is composed of several $\mathrm{V}$-shape regions, called Arnol'd tongues (phase- or frequency-locking), originating at 1,2,3, etc. times the forcing period $T_{F}$. These regions correspond to $1: 1,2: 1,3: 1$ frequency-locking zones (3:2 and 5:2 can also be guessed). Periodic solutions are found within these regions which originate generally speaking at $T_{U L C}=(m / n) T_{F}$. No synchronisation is possible when $\gamma$ is zero but synchronisation may occur already for infinitesimally small $\gamma$. Then, for increasing $\gamma$, the synchronisation region widens and synchronisation becomes more stable up to an optimum value of the forcing. Above this optimum value, the synchronisation becomes less and less effective, because at large $\gamma$ the system is too much steered away from its natural dynamics; it may even be driven into chaos at yet higher forcing amplitude (Mettin et al. 1993) but this case is beyond our focus.

In order to perform an accurate validation of the synchronisation region given by the LLE $\left(\lambda_{\max }<0\right)$ method, we computed the main Arnol'd tongues boundaries with the more accurate numerical continuation methods such as AUTO (Doedel et al. 2009). The case of periodic forcing $[F(t)=\sin (\omega t)]$ with $\beta=0$ has been already extensively studied in the literature, analytically assuming some approximations (Guckenheimer and Holmes 1983, pp. 70-75), and using numerical algorithms for pseudo-arc length continuation (Mettin et al. 1993). The usual approach extends the original nonautonomous system by additional differential equations for the forcing so that the system becomes autonomous, and then explores the $(\omega, \gamma)$ parameter space. In this way, we computed Arnol'd tongue boundaries as saddle-node of limit cycle bifurcations for the extended system with $\alpha=11.11$ and $\beta=0.25$. Note that the asymmetry introduced here with the parameter $\beta$ adds slightly more complexity and induces additional features to the diagrams documented in these papers.

Superposition of LLE calculations and bifurcation boundaries in Fig. 6a shows that the synchronisation regions obtained with the two different techniques match perfectly. This is a confirmation that the method based on the LLE works fine and we will be able to use it for the case of the quasiperiodic insolation forcing. Note that bifurcation boundaries are also drawn in Fig. $6 \mathrm{c}$ in order to stress the correspondence with yet another method of detecting synchronisation that will be discussed in Sect. 4 .

\subsection{Astronomical quasiperiodic forcing}

For the case of the quasiperiodic insolation forcing (Fig. 6b), the region of synchronisation appears to be in one single piece with some indications of tongues (mode locking) at small $\gamma$. These tongues are in fact well-separated, as it can be even more clearly seen on Fig. $6 \mathrm{~d}$ which is of higher resolution.

In other words, whatever the value of the natural period $T_{U L C}$ of the paleoclimatic system, it has a higher probability of being synchronised onto the insolation forcing.

\section{Non uniqueness: multistability and basins of attraction}

The detection of synchronisation using the LLE $\left(\lambda_{\max }<0\right)$ gives only a Yes/No-type of information (i.e. synchronisation inside the tongues, no synchronisation outside), without making any distinction between different tongues as this would require information about multistability. For example, Fig. $6 \mathrm{~b}$ indicates synchronisation for the parameter settings marked with the symbol ' $x$ ' but gives no information about the corresponding number of attracting trajectories (we know that there are three different attracting trajectories in that case, from Fig. 5e). To explore the problem of multistable synchronisation, we propose a clustering method that not only allows us to detect synchronisation, but additionally provides information about the number of attracting trajectories denoted here with $N$. 

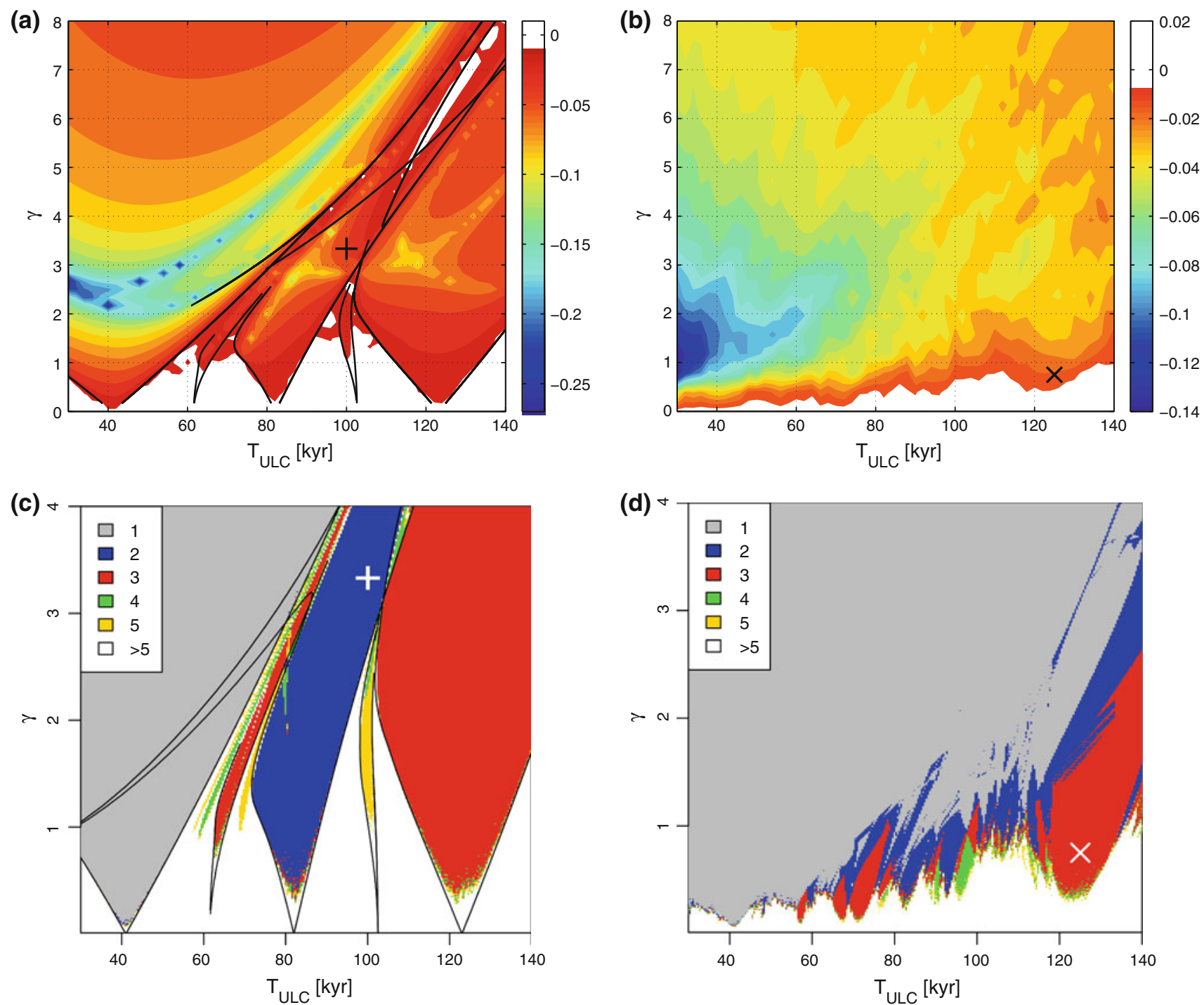

Fig. 6 Detection of synchronisation by two different methods: the largest Lyapunov exponent $\left(\lambda_{\max }\left[\mathrm{kyr}^{-1}\right]<0\right)($ top $)$ and the numerical estimate of the number of attracting trajectories $N$ by a clustering technique (bottom), and for two different types of forcing: a 41-kyr purely periodic forcing (left) and the quasiperiodic insolation forcing given by Eq. 1 (right). These diagrams show for which values of the parameters $\left\{T_{U L C}, \gamma\right\}$ synchronisation of the climate system occurs for the ice age model Eqs. 4a, 4b with $\alpha=11.11, \beta=0.25$, and $\tau=35.09$. For the 41-kyr periodic forcing case, the bifurcation boundaries of the Arnol'd tongues obtained with the more accurate numerical continuation method AUTO are superimposed, for validation purposes (black curve), and match perfectly. The symbol ' + ' refers to the specific $A T s$ illustrated in Fig. $5 \mathrm{c}$, for which $N=2$. The symbol ' $x$ ' refers to the specific ATs illustrated in Fig. 5e, for which $N=3$. a The region with $\lambda_{\max }<0$ corresponds to synchronisation;

4.1 Multistability analysis: numerical estimate

of the number of attracting trajectories

$N$ by a clustering technique

Consider the case of the quasiperiodic insolation forcing with the three attracting trajectories, i.e., $N=3$ (Fig. 5e).

we recognize its underlying Arnol'd tongue structure. b The broad region of synchronisation appears to be in one single piece with some indications of well-separated tongues (mode locking) at small $\gamma$, which is a typical signature of the quasiperiodic insolation. c The region inside the synchronisation tongues is now coloured in function of $N$. In practice, small positive values of $N$ correspond to synchronisation onto a few attracting trajectories, while high values indicate no synchronisation. For the tongue corresponding to a frequency-locking $n: 1$, we have $n$ attracting trajectories. d The structure of the synchronisation zone is much more complex, consisting of intermingled series of Arnol'd tongues. The region with one attracting trajectory, corresponding to unique or monostable generalised synchronisation, is the largest. However, there are also parameter sets with $N=2,3$ or even more attracting trajectories

Although $N$ can often be easily assessed visually, we want to automatically detect and count the number of $A T \mathrm{~s}$ (attracting climatic trajectories). As a matter of fact, $N$ can be easily estimated in the following way. Fix a time $t$ that defines a two-dimensional $(x, y)$-section in the $(x, y, t)$ phase space. Then start with a grid of initial conditions at 


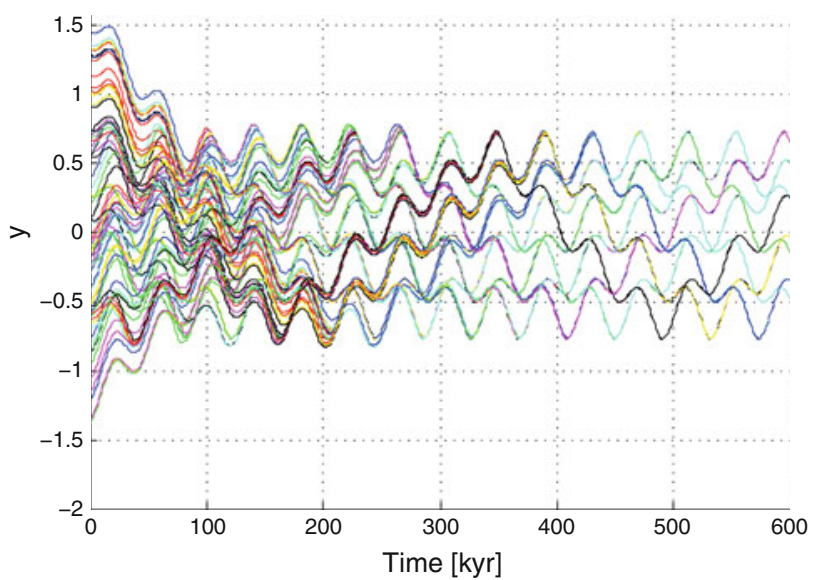

Fig. 7 Illustration of the importance of choosing the time interval $t-t_{0}$ large enough so that the transient behaviour is gone, in order to have sufficiently well formed clusters. Here, choosing $t=550 \mathrm{kyr}$ ensures that the eight clusters are already formed, starting from $t_{0}=0$

some time $t_{0}<t$ and take $t-t_{0}$ sufficiently large so that all the initial conditions converge to the attracting trajectories at $t$. Since each $A T$ is represented by a point on the $(x, y)$-section, the problem of counting attracting trajectories reduces to a simple clustering problem. We designed a suitable automatic cluster detection algorithm that counts the number of clusters to obtain an estimate of $N$. For example, Fig. $5 \mathrm{f}$ shows ${ }^{6}$ the $(x, y)$-section of the threedimensional $(x, y, t)$ phase space at $t=550 \mathrm{kyr}$, given 70 initial conditions at $t_{0}=0$. The 70 trajectories converge onto three (highly concentrated) clusters corresponding to the three attracting trajectories.

The idea of using cluster analysis for paleoclimatic dynamics comes from the natural fact that clustering is another way of looking at generalised synchronisation where negative LLE makes the trajectories cluster more efficiently. This provides another insightful viewpoint on the problem of identification of the number of synchronised solutions of the paleoclimatic system: the more stable the synchronisation, the more efficient the formation of clusters.

Two important aspects of cluster analysis ${ }^{7}$ have to be considered to avoid risks of mis-identification of clusters:

\footnotetext{
${ }^{6}$ See also Fig. 18 for a detailed view.

${ }^{7}$ Cluster analysis or clustering is the assignment of a set of observations into subsets (called clusters) so that observations in the same cluster are similar in some sense. This is a common technique for statistical data analysis used in many fields for countless applications. There exists many types of clustering, along with several methods, among which: hierarchical, partitional, spectral, kernel PCA (principal component analysis), k-means, c-means and QT clustering algorithms.
}

- the notion of a cluster is based on the threshold distance ${ }^{8}$ $d_{T}$ that has to be carefully chosen. If $d_{T}$ is chosen too large, there will be just one cluster including all points; if it is too small, no clusters will form with more than one point.

- In order to have sufficiently well formed clusters, the time interval $t-t_{0}$ must be chosen large enough so that the transient behaviour is gone; an illustration of the convergence is given in Fig. 7.

In our system, the convergence was fast and the clusters were highly concentrated and clearly separated for the great majority of the parameters. Therefore, defining a right threshold was quite easy and not critical. However, for small values of $\gamma$, the convergence is weaker, as can be seen in the bottom of the tongues on Fig. 6c.

Depending on the type and amplitude of the forcing $\gamma$, we can have potentially a whole range of possible numbers of attracting trajectories $N$, ranging from one (Tziperman et al. 2006), to a few (two in the 41-kyr periodic forcing example in Fig. $5 c$, d, or three in the quasiperiodic insolation forcing example in Fig. 5e, f). When no forcing is considered (Fig. 5a, b), or there is forcing but no synchronisation occurs, we find no clusters at all. This means that there are as many points in the $(x, y)$-section at time $t$ as initial conditions at time $t_{0}$. Clearly, it is difficult to numerically distinguish between no synchronisation and a large number of attracting trajectories $(N \gg 1)$. Therefore, we restrict ourselves to just six different regions in Fig. 6, where we use white to indicate when there are none or more than five attracting trajectories.

Now, we apply the numerical cluster analysis in the case of the periodic forcing (Fig. 6c) and of the quasiperiodic forcing (Fig. 6d). We set $t=0$ and consider a grid of 49 initial conditions covering $x \in[-2.2,2.2]$ and $y \in$ $[-2.2,2.2]$ at the initial time $t_{0}=-40 T_{F}$ for the periodic forcing ( $T_{F}$ is the period of the forcing), and $t_{0}=$ $-1,600 \mathrm{kyr}$ for the astronomical forcing.

Two points in the $(x, y)$-section are estimated to belong to a different cluster if their Euclidean distance is greater than 0.1 .

\subsection{1-kyr periodic forcing (main obliquity term)}

An illustration of the three possible synchronised solutions $(N=3)$ existing for the 3:1 frequency-locking on a periodic forcing is given in Fig. 8, where the response can be locked on one of the periods of the forcing. More generally,

\footnotetext{
8 This threshold distance $d_{T}$ appears in any computation related to cluster analysis. It is widely used for determining neighbours in a series of different methods, like the Recurrence Plots (RP) analysis in complex networks (Marwan et al. 2009; Donges et al. 2009).
} 


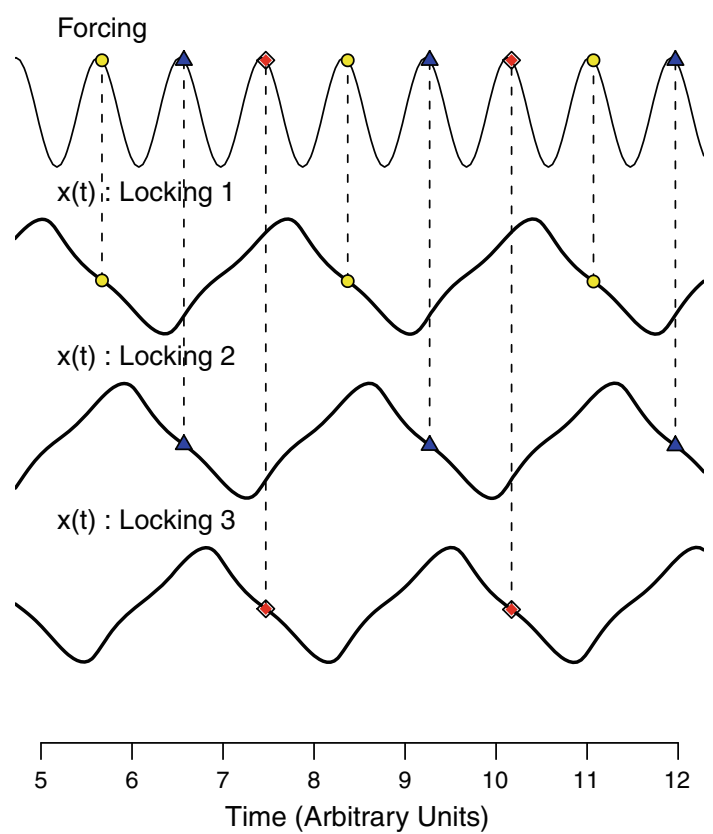

Fig. 8 Illustration of the three possible synchronised solutions $(N=3)$ existing for the frequency-locking 3:1 on a purely periodic forcing, in the time series format. The response can be locked on one of the periods of the forcing

$N$ corresponds to the number of forcing cycles associated with the synchronisation regime $(N=1$ for $1: 1 ; N=2$ for $2: 1 ; N=3$ for $3: 2,3: 1$, etc.). ${ }^{9}$ The resulting pattern of different $N$ is in agreement with the bifurcation diagram (Fig. 6c). For example, $N=3$ in the 3:1 tongue. This method allows one to visualise the $4: 3$ tongue $(N=4)$ around $41 \times 4 / 3 \approx 54.67 \mathrm{kyr}$, and even the 5:4 tongue $(N=5)$ around $41 \times 5 / 4 \approx 51.25 \mathrm{kyr}$, both to the left of the $3: 2$ tongue (around $41 \times 3 / 2 \approx 61.5 \mathrm{kyr}$ ). It is also seen that $N$ is generally larger where different synchronisation regimes coexist; this is the case between the $2: 1$ and $3: 1$ regimes around $\gamma=4$.

\subsection{Astronomical quasiperiodic forcing}

Figure 6d shows that synchronisation occurs for most parameter configurations. The region with one attracting trajectory $(N=1)$, corresponding to unique or monostable generalised synchronisation (Rulkov et al. 1995), is the largest. However, there are also parameter sets with $N=2$, 3 or even more attracting trajectories. They indicate $m u l$ tistable generalised synchronisation where different possible stable relationships (2) between the forcing and the oscillator response coexist.

\footnotetext{
9 This statement relies on the system invariance with respect to a time-shift of one forcing period (Tziperman et al. 2006 show a very nice illustration of this point).
}

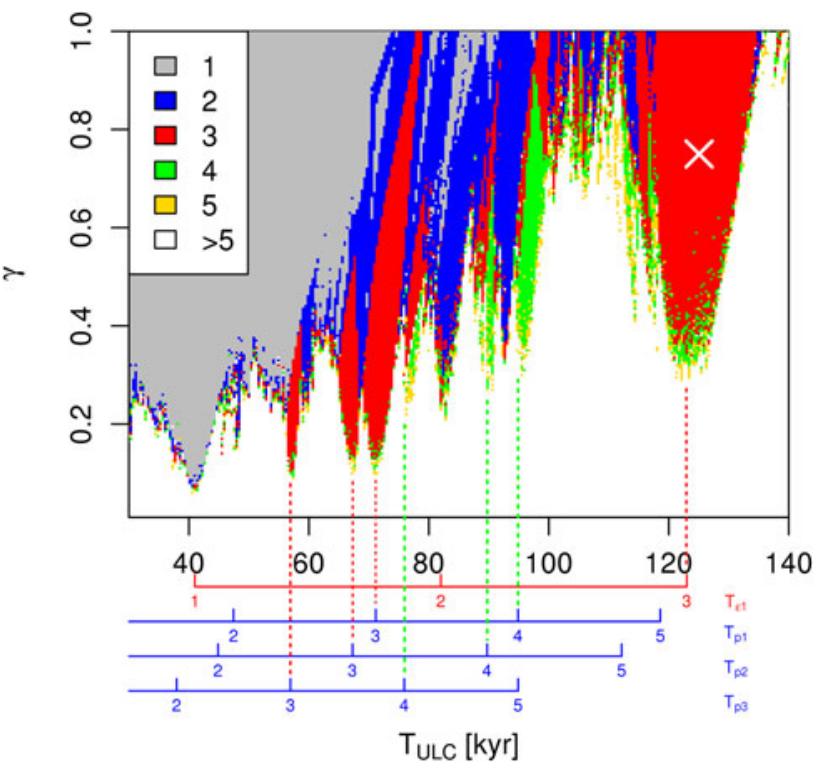

Fig. 9 A careful examination of the lower part (low $\gamma$ ) of Fig. 6d allows an insightful physical interpretation. The underlying structure of the intermingled series of Arnol'd tongues can be understood: it is a mixing of several Arnol'd tongues series corresponding to each of the main components of the spectrum of the astronomical forcing, yielding a resulting pattern which looks like misaligned combs. Subscales based on the period of the four main components $\left(\epsilon_{1}, p_{1}, p_{2}\right.$ and $p_{3}$ ) of the insolation highlighted in Fig. 2 allows a clear recognition of each individual Arnol'd tongues series

An expanded view of Fig. 6d in the lower values of $\gamma$ is given in Fig. 9, which allows an insightful physical interpretation. Three tongues with $N=1,2,3$ are rooted at $T_{U L C} / T_{\epsilon_{1}}=1,2$ and 3 , respectively, suggesting a synchronisation on the main obliquity component of the astronomical forcing of the same nature as synchronisation on a periodic forcing. A series of other synchronisation tongues with $N>1$ appear; they correspond to $2: 1$ $(N=2), 3: 1(N=3), 4: 1(N=4)$ and even 5:1 $(N=5)$ synchronisation on the three leading components of precession, denoted $p_{1}, p_{2}$ and $p_{3}$ (see Fig. 2). Consequently, the richness of the astronomical forcing effectively widens the parameter range for which synchronisation occurs, compared to a periodic forcing. The phenomenon may be understood intuitively: just as you are more likely to tune on some radio station if you are surrounded by a dozen of free FM emitters, the system is more likely to synchronise on the rich astronomical forcing than on a periodic forcing. Synchronisation with $N=1$ or 2 , found for larger $\gamma$, can be interpreted as a form of combined synchronisation on both obliquity and precession.

It is crucial to appreciate that synchronised solutions are not periodic and that, unlike in the periodic forcing case, different synchronised solutions for a given set of parameters are not time-shifted versions of each other. The idea that different synchronised solutions coexist is of practical 


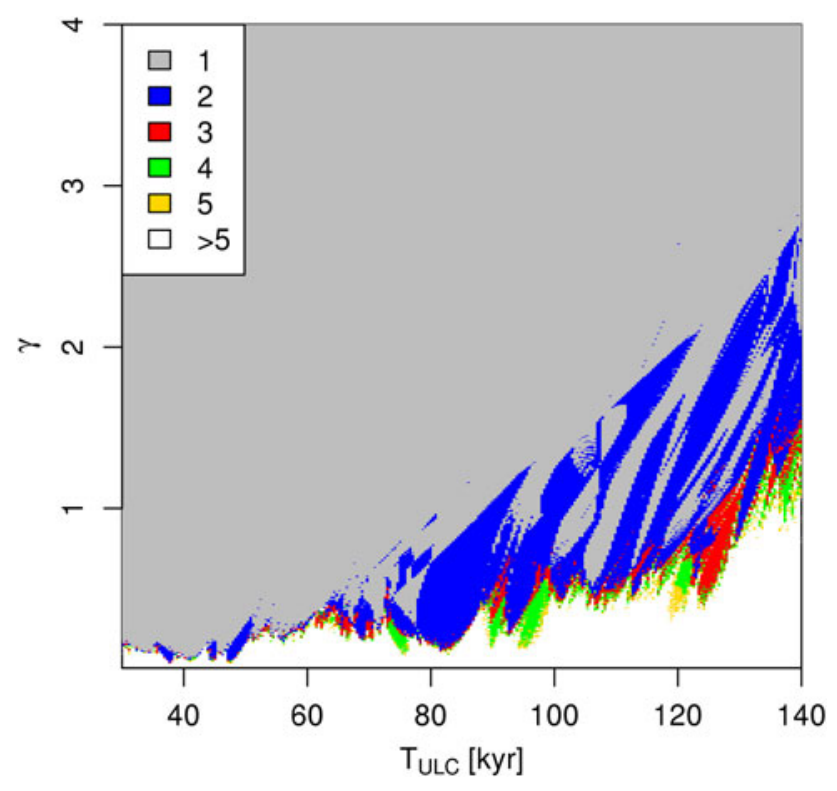

Fig. 10 Same as Fig. 6d, but now with $\beta=0.6$ instead of $\beta=0.25$. The particular values of $N$ change, as the pattern of intermingled tongue series is different, but the main conclusion about the multistability remains

relevance for paleoclimate theory. Namely, the set of parameters used to obtain the fit to the paleoclimatic records shown in Fig. 3 give two distinct solutions at $t=0$ when started from a grid of initial conditions at $t_{0}=$ $-700 \mathrm{kyr}$. Sensitivity studies show that the choice of $t-t_{0}$ is sometimes important for estimating correctly $N$. However, tests with $t-t_{0}$ as large as $200 \mathrm{Myr}$ of astronomical time suggest that several attracting trajectories may coexist at the asymptotic limit of $t_{0} \rightarrow-\infty$.

A similar numerical cluster analysis plot for $\beta=0.6$ is shown in Fig. 10 in order to give an idea of the effect of this parameter (a more detailed analysis is performed in Sect. 5). The main conclusion about the multistability remains, but the particular values of $N$ change, as the intermingled tongue series are different.

\subsection{Evolving geometry of the basins of attraction}

Each $A T_{i}(i=1 \ldots N)$ has its own basin of attraction ${ }^{10}$ (Barnes and Grimshaw 1997), that is defined as the set of all initial conditions in the $(x, y, t)$ phase space that converge to that $A T_{i}$ as time tends to infinity. For our nonautonomous system Eqs. 4a, 4b, we can study basins of attraction in the $(x, y)$-section for different but fixed values of initial time $t_{0}$, and observe how they vary with $t_{0}$. A given initial condition at time $t_{0}$ lies in the basin of

\footnotetext{
10 A more formal definition of the basin of attraction for nonautonomous dynamical systems is given in Kloeden (2000) and Langa et al. (2002).
}

attraction of $A T_{i}$ if it approaches $A T_{i}$ as time tends to infinity. The whole phase space can then be 'painted' with several colours, each colour representing a specific basin. Technical details about the computation of the basins of attraction by use of the specific classification algorithm developed (see Fig. 18) are given in "Appendix 3". Basins of attraction are of major importance because they provide the information about global or nonlinear stability of synchronisation. If we care about predictability, basin boundaries indicate when a change in the attracting climatic history is likely.

The evolving geometry of the basins of attraction is shown in Figs. 11 and 12, for the case of 41-kyr periodic forcing and the quasiperiodic astronomical forcing, respectively. The evolution is shown as a comic strip, where each subfigure has an $x$-axis ranging from -1.5 to 1.5 , and a $y$-axis ranging from -2.5 to 2.5 (the axis labels have been removed for a better readability).

In the case of a periodic forcing (two basins), the pattern repeats itself periodically (compare the $t_{0}=0 \mathrm{kyr}$ to the $t_{0}=40 \mathrm{kyr}$, and to the $t_{0}=80 \mathrm{kyr}$ subfigures in Fig. 11). However, in the case of the quasiperiodic forcing (three basins), the pattern is much more intricate and seems not to repeat itself for the time horizon considered here, cf. Fig. 12.

The ratio between the area of a basin of attraction and the considered area of the phase space can be interpreted as a probability to converge to the corresponding attracting trajectory when starting from a randomly chosen initial condition. In the case of the periodic forcing, the two ATs are roughly equally likely for all $t_{0}$ as could be guessed from Fig. 5c. However, this is not the case for the quasiperiodic forcing where the probability to reach the same attracting trajectory may vary significantly in time. For example, the yellow basin is rather small at $t_{0}=0 \mathrm{kyr}$ but becomes much larger at a later time $t_{0}=90 \mathrm{kyr}$.

In the multistable regime, if an $A T_{i}$ happens to lie sufficiently close to its basin boundary, then small perturbations could make the climate jump to another (coexisting) $A T_{j \neq i}$, reducing predictability. This phenomenon is illustrated in Sect. 6.

\section{Influence of the symmetry-breaking parameter $\beta$}

As the parameter $\beta$ controls the asymmetry ${ }^{11}$ of the glaciation/deglaciation sawtooth structure (a higher value of $\beta$

\footnotetext{
$\overline{11}$ A variation of the parameter $\beta$ may also imply a very fast transition from a small amplitude limit cycle to a large amplitude relaxation cycle, explained by the so-called Canard phenomenon, cycles, and explosion (Benoît et al. 1981; Guckenheimer et al. 2000; Guckenheimer and Haiduc 2005).
} 

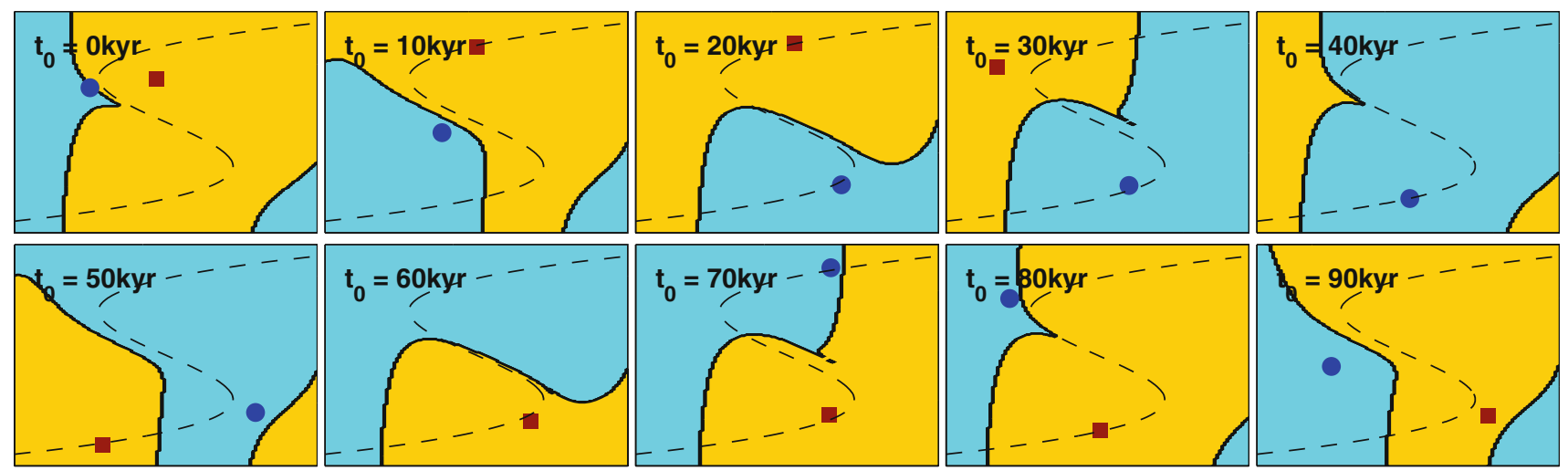

Fig. 11 Evolving geometry of the two basins of attraction for system Eqs. 4a, 4b, with $\alpha=11.11, \beta=0.25, \gamma=3.33$ and $\tau=35.09$. Case of a 41-kyr periodic forcing: the pattern is 82-kyr periodic (frequency-locking 2:1). The function $\Phi^{\prime}(y)=y^{3} / 3-y=x$,

corresponding to the slow manifold, is also shown (dashed curve). The attracting trajectories (symbols) sometimes lie close to the boundary of their own basin of attraction. Note that one $A T$ is missing on the 40 and $60 \mathrm{kyr}$ panels, as it stands out of the range of the plot
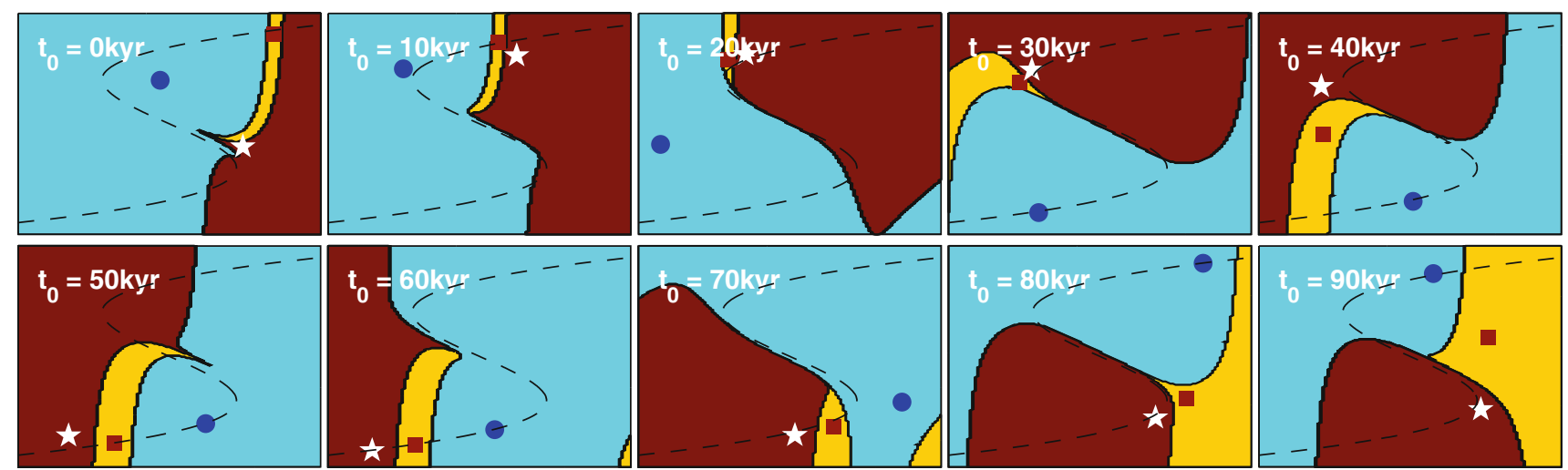

Fig. 12 As in Fig. 11, but now for the quasiperiodic insolation forcing case, with $\gamma=0.12$. For this choice of parameters, there are three basins of attraction. The pattern is quasiperiodic, constituting the specific signature of the insolation

(a)

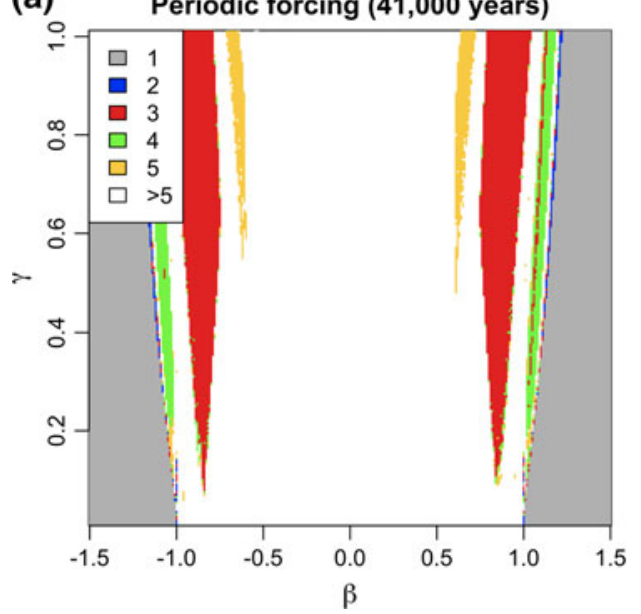

Fig. 13 Numerical estimate of the number of attracting trajectories $N$ plotted as a function of the asymmetry parameter $\beta$ and the amplitude of the forcing $\gamma$ for the ice age model Eqs. 4a, 4b with $\alpha=11.11$ and $\tau=35.09$, assuming $\mathbf{a} 41-\mathrm{kyr}$ periodic forcing and $\mathbf{b}$ the quasiperiodic astronomical forcing given by Eq. 1. Synchronisation occurs in a larger (b)

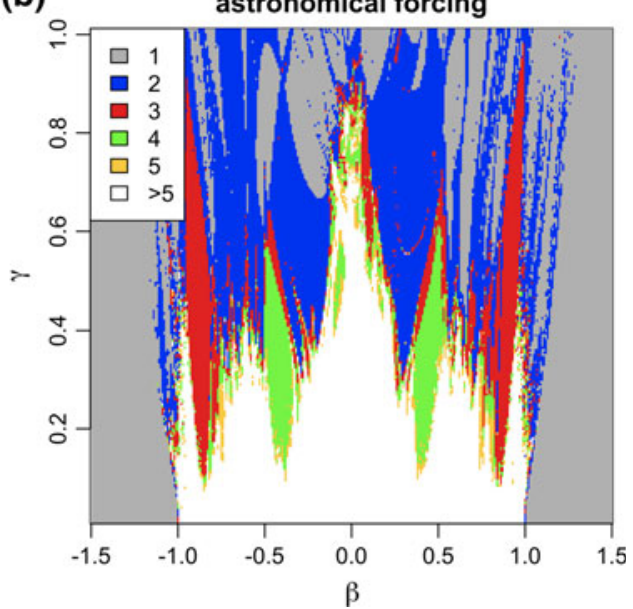

area of the parameter space in response to the astronomical forcing than in response to the periodic forcing, and the pattern of different $N$ for larger $\gamma$ is much richer and more complex; this structure emerges from the interaction with different harmonics of the astronomical spectrum and their beatnotes 
leads to an enhanced asymmetry), it is useful to investigate its effect. We have already indicated in Fig. 10 that multistability depends on $\beta$ in the case of the quasiperiodic insolation forcing. A more systematic approach encompassing the whole range of $\beta$ is shown in Fig. 13a, 13b, for the case of 41-kyr periodic forcing and the quasiperiodic astronomical forcing case, respectively.

First consider the 41-kyr periodic forcing (Fig. 13a). To understand this figure, recall that the unforced oscillator (i.e., $\gamma=0$ ) has a stable fixed point for $|\beta|>1$ and a stable limit cycle for $|\beta|<1$.

The system responds almost linearly to the forcing when $|\beta|$ is sufficiently large. This explains regions of unique synchronisation $(N=1)$ where only one climate response is possible. The system becomes excitable when $|\beta|$ is just slightly greater than one. If the forcing is large enough it will excite oscillations. In this case, $N$ is equal to the number of initial conditions if synchronisation is lost, or to a smaller number if synchronisation occurs.

Consider now the interval $-1<\beta<1$. For this, keep in mind (1) that the period of the unforced oscillation $T_{U L C}$ varies by almost a factor of two within the range $0<|\beta|<1$, and (2) that synchronisation requires some relation between the period of the unforced oscillations and the forcing period. Consequently, synchronisation on the periodic forcing occurs only for fairly narrow ranges of $\beta$ that are symmetric around zero. The figure reminds us of Arnol'd tongues. The main synchronisation regimes detected here correspond to 4:1, 3:1 and 5:2 frequency-locking. Outside these synchronisation regimes, the system fails to converge to a sufficiently small set of attracting trajectories, meaning that the forcing is not an efficient pacemaker.

Even if not obvious, Fig. 13a can be partly explained by considering Fig. 6c. The multistability plot can indeed be considered here as being 3-dimensional in the parameter space $\left\{T_{U L C}, \gamma, \beta\right\}$, but the intricate aspect is that Figs. 13a and $6 \mathrm{c}$ are not straight cuts into this 3 -dimensional space, as the relation between $\beta$ and $T_{U L C}$ is not linear. For example, if you take the parameters of Fig. 13a, i.e. $\tau=35.09$, it corresponds to $T_{U L C}=100 \mathrm{kyr}$; so, when performing the cut in Fig. 6c the yellow region will be reached in the upper part (pay attention that the $y$-scale is different), what is consistent with what is found in Fig. 13a for low $|\beta|$ values. Then, when increasing $|\beta|, T_{U L C}$ increases, i.e. one moves a bit on the right on Fig. $6 \mathrm{c}$, reaching then the red region, which is again consistent with what is found in Fig. 13a for higher $|\beta|$ values. For $|\beta|>1$, Fig. $6 \mathrm{c}$ is no longer relevant. To have a deeper understanding, much more views in the 3-dimensional space would be required (e.g. Fig. 6c should be done for several values of $\beta$ ), but it is not the goal of this paper.

Finally, compare the 41-kyr periodic forcing situation with that obtained with the astronomical forcing (Fig. 13b).
Synchronisation now occurs in a larger area of the parameter space. Whereas the structure of the periodic forcing is preserved as long as the forcing amplitude is low enough, there is a much richer and more complex pattern of different $N$ for larger $\gamma$. This pattern emerges from the interaction with different harmonics and their beatnotes.

Note that Fig. 13a, b have a very high level of symmetry with respect to $\beta$, which was expected as the system Eqs. $4 \mathrm{a}, 4 \mathrm{~b}$ is invariant under the transformation $\{x, y, \beta, F\} \rightarrow\{-x,-y,-\beta,-F\}$.

\section{Robustness of synchronisation}

Robustness or reliability of synchronisation can be studied in terms of two properties of an attracting climatic trajectory. Local stability analysis based on the short-term LLE $\left(\lambda_{\text {max }}^{H}\right)$ provides information about the short-term local convergence towards the $A T$. For example, a temporary loss of local stability indicated by $\lambda_{\max }^{H}>0$ will cause a temporary loss of synchrony and divergence from the $A T$ even though the trajectory is stable on average $\left(\lambda_{\max }<0\right)$. Global stability analysis based on the geometry of the basins of attraction for different ATs provides information about the system's response to external perturbations such as random fluctuations. For example, an external perturbation may push a climatic trajectory outside of its basin of attraction. Robustness and uniqueness of synchronisation become closely linked when there are coexisting attracting trajectories. Robustness is compromised most when a temporary loss of local stability coalesces with a weakening of the global stability. We will now briefly discuss these two effects that could restrict the prediction horizon for the evolution of climatic trajectories.

\subsection{Temporary desynchronisation via loss of local stability}

Some additional experiments made in our paleoclimatic framework reveal another strange behaviour in the system Eqs. 4a, 4b, that can be deduced from a careful inspection of Fig. 14. In the presence of small additive noise, we notice that nearby trajectories could diverge for some time, like those around $t \approx 157 \mathrm{kyr}$.

Such temporary divergence is similar to desynchronisation bursts (Rulkov et al. 1995) and strongly suggests to investigate the evolving sign of the short-term LLE $\lambda_{\text {max }}^{H}$ along the attracting climatic trajectory. We computed $\lambda_{\max }^{H=50 \mathrm{kyr}}$ along one of the two attracting trajectories of system Eqs. 4a, 4b, subject to insolation forcing given by Eq. 1. The value $H=50 \mathrm{kyr}$ has been chosen because it corresponds to a reasonable short-term timescale with respect to the length of the glacial/interglacial cycles. The 


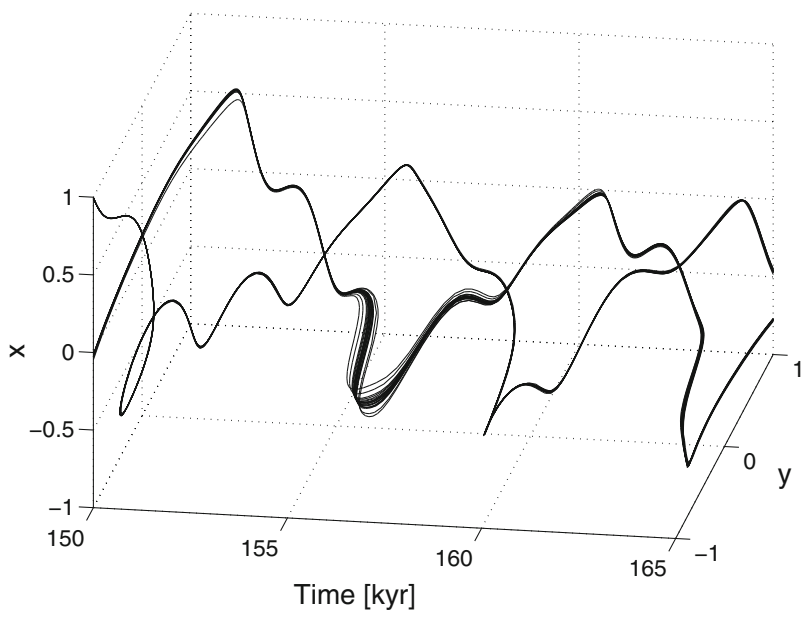

Fig. 14 The temporary divergence of nearby climatic trajectories reveals short-term local instabilities (e.g. around $t \approx 157 \mathrm{kyr}$ ). This illustration was obtained by considering a set of 50 random initial conditions at $t_{0}=0$ within $x \times y \in[-1,1] \times[-1,1]$, and by adding some noise of small amplitude (i.e. $b=0.1$ in the equation $d y=$ $-\tau^{-1}\left(\alpha\left(\Phi^{\prime}(y)-x\right)\right) d t+b d W$, with $W$ a Wiener-process $)$ in the system at every time step in order to trigger the instabilities. The model used is Eqs. 4a, 4b with $\alpha=11.11, \beta=0.25, \gamma=0.39$ (quasiperiodic forcing), and $\tau=3.33$

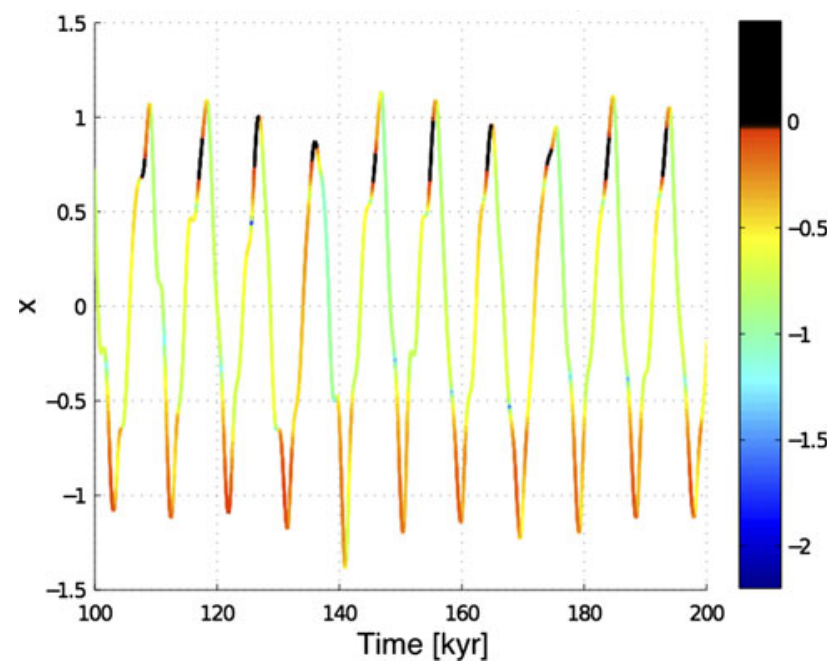

Fig. 15 Short-term largest Lyapunov exponent $\lambda_{\max }^{H=50 \mathrm{kyr}}$ of one of the two attracting trajectories of system Eqs. $4 \mathrm{a}, 4 \mathrm{~b}$, subject to insolation forcing given by Eq. 1, and for the same parameters as in Fig. 14. The attracting climatic trajectory has been coloured according to the values of $\lambda_{\max }^{H=50 \mathrm{kyr}}$, revealing temporary desynchronisation; this explains the divergence of nearby trajectories found in Fig. 14, e.g. around $t \approx 157 \mathrm{kyr}$

result is shown in Fig. 15, where the attracting climatic trajectory has been coloured according to the values of $\lambda_{\max }^{H=50 \mathrm{kyr}}$. Although the system is synchronised on a long term $\left(\lambda_{\max }=-0.2 \mathrm{kyr}^{-1}\right.$, see Fig. 17), we see here that there exist episodes with positive values of the short-term LLE $\lambda_{\max }^{H}$, revealing temporary desynchronisation
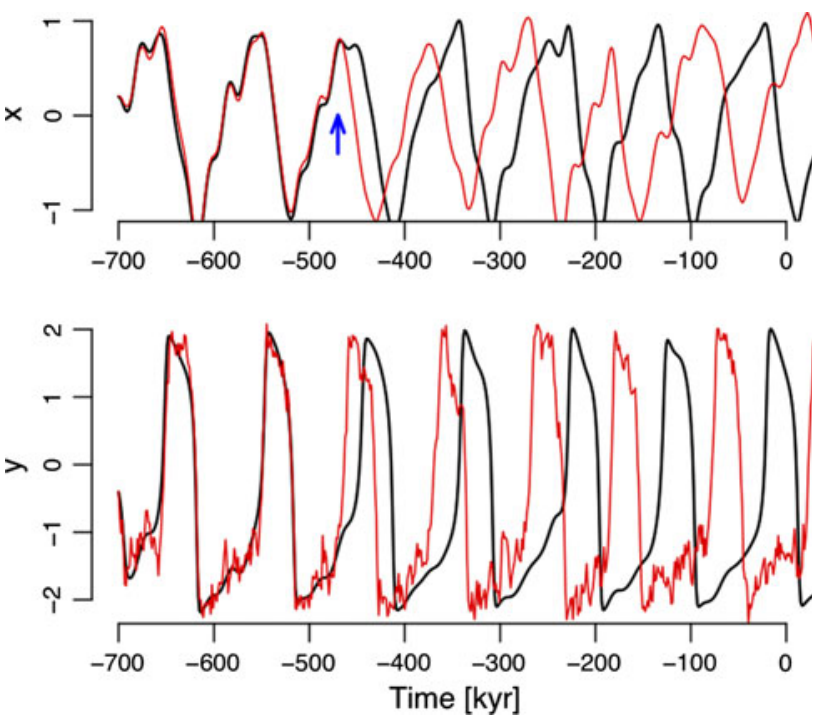

Fig. 16 Sensitivity of the climatic system to perturbations. The same solution of the ice ages model Eqs. $4 \mathrm{a}, 4 \mathrm{~b}$ as in Fig. 3 is plotted (black) along with one sample trajectory of the same system (red), but with additive fluctuations added to the fast variable: $d y=$ $-\tau^{-1}\left(\alpha\left(\Phi^{\prime}(y)-x\right)\right) d t+b d W$, with $b=0.5 \sqrt{\omega_{\epsilon_{1}}}$ and $W$ a Wienerprocess. The arrow shows the time of the jumping from one to the other climatic attracting trajectory, which reduces the predictability of the timing of the ice ages

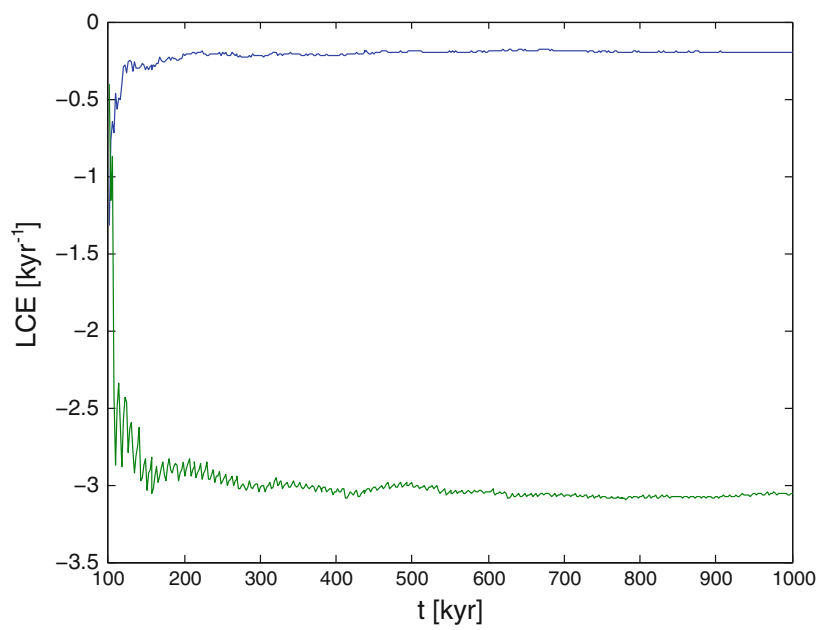

Fig. 17 Convergence of the two LCEs of one of the two attracting trajectories of the system Eqs. 4a, 4b, subject to the insolation (Eq. 1) with $\gamma=0.39$; some transient is skipped. For $\tau=3.33$, we have $\lambda_{\text {max }}=-0.2 \mathrm{kyr}^{-1}<0$ (for $\tau=35.09$, the value must be scaled accordingly, which would give $-0.019 \mathrm{kyr}^{-1}$ ), so the system is synchronised on a long term

(Wieczorek 2009). This explains the divergence of nearby trajectories found in Fig. 14.

These results remain unchanged with respect to the most important parameters of the model. For example, our main conclusion about the stability remain qualitatively valid, even for different values of $\alpha$ (like $\alpha=100$ ), or with a different type of potential $\left(\Phi_{5}^{\prime}(y)=(y+1.7)(y+1.58)\right.$ 


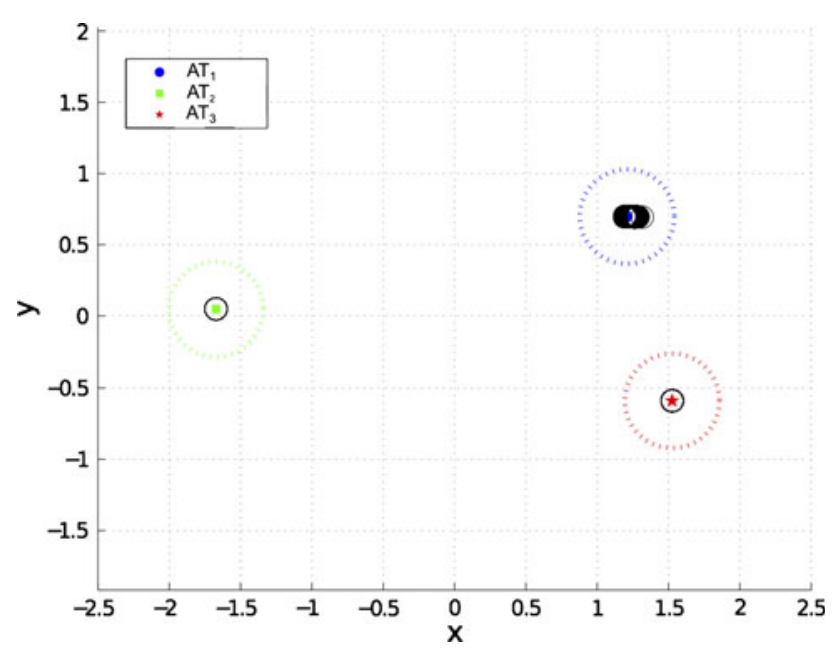

Fig. 18 Illustration of the classification algorithm, which allows to compute the basins of attractions. The position of the 70 trajectories (black circles) with respect to the three attracting trajectories (symbols) at time $t=550 \mathrm{kyr}$, starting from $t_{0}=20 \mathrm{kyr}$, are shown. If the trajectory falls into a dotted circle, classification is possible. The 70 trajectories form three highly concentrated clusters, corresponding to the three attracting trajectories

$(y+0.8)(y)(y-0.5))$, even if the shape and size of the limit cycle and the boundaries of the basins of attraction are of course different. The effect of the insolation function $F(t)$ has also been checked: we compared the attracting trajectories for the insolation given by Eq. 1 to those for the insolation given by Laskar et al. (2004). As these insolation functions are very similar, the results are also very similar, and no difference was noticed.

At first glance, it may appear that these episodes of temporary divergence are not relevant to the robustness of synchronisation because climatic trajectories converge back to the attracting trajectory on a long term. However, other effects may be present that could strongly amplify such temporary divergence. They are identified below.

\subsection{Sensitivity to perturbations: preliminary results}

Consider again Fig. 12 showing $(x, y)$-sections with coexisting attracting trajectories in the case of the quasiperiodic insolation, and their basins of attraction for different values of $t_{0}$. Suppose now that the system is subject to additive fluctuations (for example, these may represent volcanic eruptions). Under certain conditions, such external perturbations may cause a displacement of the trajectory to a different basin of attraction, causing a jump ${ }^{12}$ to another attracting trajectory.

As a further illustration of this idea we show in Fig. 16 two attracting trajectories (in the time series format) that

\footnotetext{
12 In the periodic forcing case the phenomenon of jumping from one attracting trajectory to another in response to a perturbation is called a phase slip (Pikovsky et al. 2001, p. 238).
}

coexist for the same system parameters as those used for the fit of Fig. 3, but with additive fluctuations added to the fast variable (see legend for details). A jump from one trajectory to another at around $-475 \mathrm{kyr}$ (arrow) may clearly be identified. This jump suggests that a climatic trajectory is robust against fluctuations if it stays away from the basin boundary but its robustness can weaken significantly due to the weakening of the global stability near the basin boundary.

Figure 15 indicates the exact location where the "synchronisation jumps" are likely to occur. At first glance, it seems that the most critical times are those corresponding to large positive values of $x$ (i.e. to large ice volumes according to Fig. 3). More precisely, these critical times are actually 'just before' the maximum ice volume, and the jump occurs then at the beginning of the deglaciation. This is consistent with the stochastic realisation shown in Fig. 16.

We conjecture that externally triggered jumps between coexisting attracting climatic trajectories are most likely when the temporary desynchronisation due to the loss of local stability coalesces with the weakening of the global stability due to the proximity to the basin boundary.

Note finally that a related result has been indicated by Paillard (2001, on Fig. 14), where the model has only one attracting trajectory, but this $A T$ is very sensitive to changes in the model parameters. The end result is that very different trajectories for the ice volume evolution emerge from small parameter changes (in Paillard 2001, two possible trajectories depending on a change in the parameter $i_{0}$ representing an insolation threshold).

\section{Conclusions}

Previous studies have shown that locking mechanisms could be found in the ice ages problem (Le Treut and Ghil 1983; Hyde and Peltier 1985; Paillard 1998; Gildor and Tziperman 2000), but most of the time, the conclusions rely on a few particular realisations of the models, without providing a global analysis of the synchronisation phenomenon, like the one provided in this study.

Also, despite some attempts, the actual type of synchronisation has not been clearly identified or demonstrated to date. For example, (Ashkenazy 2006; Tziperman et al. 2006) speak of "nonlinear phase-locking" although they do not define suitable "phase variables" that can be used to demonstrate a fixed-in-time relationship between phases of the forcing and the oscillator response.

In this paper, we have for the first time identified, illustrated, and provided a systematic study of the phenomenon of generalised and multistable synchronisation between a simple conceptual model of the climatic glacial/ 
interglacial oscillations and the astronomical forcing. A van der Pol-type relaxation oscillator, designed to reproduce the slow-fast dynamics of the paleoclimatic records, has been used for illustration purposes, but the methodology proposed may of course be applied to other paleoclimatic models.

The dynamical systems approach proposed herein (1) allows for stability analysis of such synchronisation, (2) uncovers interesting effects related to the robustness of the synchronisation with respect to external perturbations, and (3) uncovers the phenomenon of multistable synchronisation that has been overlooked by previous studies. We have shown that, in contrast to claims in Tziperman et al. (2006), synchronisation needs not be unique.

To study the uniqueness of synchronisation, we proposed a convenient concept of the number of attracting trajectories in the phase space of the nonautonomous forced system, each of which corresponds to a synchronised solution. We computed the number of synchronised solutions using a numerical clustering technique, and uncovered that in addition to a unique or monostable synchronisation, there are parameter settings where one finds a nonunique or multistable synchronisation. At low forcing amplitude we found regions of mode locking where the system synchronises on the individual components of the astronomical forcing in a way that is similar to frequency-locking on periodic forcing (Arnol'd tongues), giving rise to coexisting synchronised solutions. As the forcing amplitude is increased, the combined effects of precession and obliquity restrict the number of possible synchronised solutions. The emerging stability diagram consists of a large region of monostable synchronisation mixed with smaller regions of multistable synchronisation. A comparison with periodic forcing shows that the system finds it easier to synchronise to quasiperiodic insolation forcing. It is therefore conceivable that the climate system wandered throughout preferential synchronisation regimes on obliquity, precession, or combinations of both, all over the history of the Pleistocene.

The robustness of generalised synchronisation was investigated in terms of the key indicators of stability of synchronised solutions: the long- and short-term largest Lyapunov exponent (local stability), and the geometry of the basins of attraction (global stability). We found that even though the synchronised solutions are locally stable on a long term, there exist episodes where the short-term largest Lyapunov exponent becomes positive, leading to temporary desynchronisation. As a result, climatic trajectories could diverge from the synchronised solution for some short period of time (it is shown here for $50 \mathrm{kyr}$ ). Moreover, we computed the evolving geometry of the basins of attraction for the coexisting synchronised solutions, and uncovered that these solutions sometimes approach the basin boundary where they become very susceptible to external perturbations. As a result, a small perturbation could make the climate jump from one synchronised solution to another, reducing predictability. We conjecture that such jumps are most likely when the temporary loss of the local stability coalesces with the proximity to the basin boundary. In this context, we briefly discussed the effect of stochastic perturbations on the timing of the ice-ages. We also illustrated the difference between the evolving geometry of the basins of attraction for periodic and quasiperiodic insolation forcing. In the case of the insolation forcing, we obtained an intricate pattern of basins of attraction that does not appear to repeat itself in time.

In this way, our results contribute to the emerging theory of predictability of ice ages. Future works will of course have to take into account the physical constraints like reproducing satisfactorily enough the so-called Mid-Pleistocene Transition.

Acknowledgments We are grateful to Guillaume Lenoir for his thorough review of several versions of the paper. The original idea of using cluster analysis for automatically identifying the number of stable locking states cropped up after presentations and discussions at the 458. WE-Heraeus-Seminar on 'SYNCLINE 2010: Synchronisation in Complex Networks', held on 26-29 May 2010 at the Physikzentrum Bad Honnef (Germany), where some preliminary results of this research have been presented in a poster. The project is funded by the ERC (European Research Council) starting grant ITOP ('Integrated Theory and Observations of the Pleistocene') under the convention ERC-StG-2009-239604. M. Crucifix is Research Associate with the Belgian National Fund of Scientific Research, and B. De Saedeleer is Postdoctoral Research Assistant with the ITOP Project. Some figures and calculations have been made with the $\mathrm{R}$ language and the Intel Fortran Compiler.

Open Access This article is distributed under the terms of the Creative Commons Attribution License which permits any use, distribution, and reproduction in any medium, provided the original author(s) and the source are credited.

\section{Appendix 1: Insolation model using 35 terms}

We give at the Table 1 the numerical values of the $3 \times 35$ terms for computing the insolation $F(t)$ following Eq. 1 in Sect. 1.

Table 1 Coefficients for the insolation model described in Eq. 1 used for the incoming solar radiation anomaly at Summer Solstice and at $65^{\circ} \mathrm{N}$

\begin{tabular}{llc}
\hline$\omega_{i}[\mathrm{rad} / \mathrm{kyr}]$ & $s_{i}\left[\mathrm{~W} / \mathrm{m}^{2}\right]$ & $c_{i}\left[\mathrm{~W} / \mathrm{m}^{2}\right]$ \\
\hline 0.153249478547167 & -11.2287376815124 & 3.51682075211241 \\
0.158148666238883 & -3.82499371467540 & -0.761851750263805 \\
0.117190147169570 & 2.28814805956066 & 1.80233702684623 \\
0.155061775112933 & -1.29770081956440 & -0.635152963728496 \\
\hline
\end{tabular}


Table 1 continued

\begin{tabular}{lcc}
\hline$\omega_{i}[\mathrm{rad} / \mathrm{kyr}]$ & $s_{i}\left[\mathrm{~W} / \mathrm{m}^{2}\right]$ & $c_{i}\left[\mathrm{~W} / \mathrm{m}^{2}\right]$ \\
\hline 0.217333905941751 & 0.380973541305497 & -1.46301711999210 \\
0.150162587421217 & 1.54904176353302 & -0.0883941912769817 \\
0.211709630908568 & -0.810768209286259 & -0.577980646565494 \\
0.156336369673117 & -0.918358442095885 & 0.196083726889428 \\
0.148350290855451 & 0.256895610735773 & -0.524697312305024 \\
0.206924898030688 & -0.335783913402678 & -0.0194792150128644 \\
0.212525165090383 & 0.267659228540196 & 0.128915417116900 \\
0.229992875969202 & 0.0696189733188958 & 0.0746231714061285 \\
0.306498957094334 & 0.0247349748169616 & 0.0140464395340974 \\
0.311398144786051 & 0.0138353727621181 & 0.0304736668840422 \\
0.004899187691716 & -0.160479848721994 & 0.0594077968934257 \\
0.264933601588513 & -15.5490493322904 & -9.70406287110532 \\
0.280151350350945 & 15.4319556361701 & 4.75247271131525 \\
0.331110950251899 & 9.0992249352734 & -10.6115244887390 \\
0.328024059125949 & -7.87065384013669 & 6.61544246063503 \\
0.326211762560183 & 0.813786144754451 & -4.52641408099246 \\
0.269742342439881 & 0.0690448504314857 & -3.31639260969558 \\
0.332923246817665 & 1.44050770785967 & 1.06339286050120 \\
0.371638925683567 & 0.925324276580528 & -1.02066758672154 \\
0.275366617473065 & 0.997628846513796 & -0.362906496840039 \\
0.323124871434233 & -0.378637986107629 & 0.527217891742183 \\
0.259396912994958 & 0.339477750517033 & -0.560509461538342 \\
0.324937167999999 & -0.576082669762308 & 1.18669572739338 \\
0.334197841377850 & 0.346906064369828 & -0.648189701487285 \\
0.274551083291250 & -0.441772417569753 & 0.289576210423804 \\
0.418183080135680 & -0.0184884064645011 & 0.109632390175297 \\
0.111684123041346 & -0.428006728186239 & 0.357006342316690 \\
0.433400828898112 & -0.0049199219454561 & -0.106148873639336 \\
0.126901871803777 & 0.257509918217341 & -0.377639794223366 \\
0.336010137943616 & -0.421809264016129 & 0.324327509437558 \\
0.177861471704732 & -0.161827722328271 & -0.362683869407858 \\
\hline & & \\
& & \\
& & \\
& & \\
& &
\end{tabular}

The first 15 terms correspond to the obliquity, while the other 20 terms correspond to the precession

\section{Appendix 2: Technical details about the calculation of the Lyapunov exponents}

We first refer the reader to the seminal papers (Shimada and Nagashima 1979; Benettin et al. 1980). The methods for computing the Lyapunov (characteristic) exponents (LCE) vary depending on the fact whether one wishes to achieve the full spectrum of the LCE (Wolf et al. 1985), or only the largest one (Rosenstein et al. 1993). Analytical derivations of the LCEs of the van der Pol oscillator do also exist (Grasman et al. 2005). In this research, we computed $\lambda_{\text {max }}$ using the standard method involving a Gram-Schmidt reorthonormalisation (GSR) of the 'tangent vectors' which is described in the review paper (Ramasubramanian and Sriram 2000). As the system Eqs. 4a, 4b and the Jacobian have an analytical form, tangent space methods (Kantz and Schreiber 2004) can be used.
We remind here the fundamental principles and tools that were used to achieve the results presented in the present paper concerning the Lyapunov exponents. Consider an $n$-dimensional continuous-time dynamical system:

$\frac{d \mathbf{Z}}{d t}=\mathbf{f}(\mathbf{Z}, t)$

where $\mathbf{Z}$ and $\mathbf{f}$ are $n$-dimensional vector fields. To determine the LCEs corresponding to some initial condition $\mathbf{Z}(0)$, we have to find the long term evolution of the axes of an infinitesimal sphere of states around $\mathbf{Z}(0)$. That is to say that we assume (Ott 2002) $\delta \mathbf{Z}(t)=$ $\delta \mathbf{Z}(0) \mathrm{e}^{\Lambda t}$, with $\Lambda=\operatorname{diag}\left(\lambda_{1}, \ldots, \lambda_{n}\right)$, where $\lambda_{i}$ are the eigenvalues of the system. For this, we consider the linearisation of Eq. 7, given by:

$\frac{d \delta \mathbf{Z}}{d t}=\mathbf{J} \delta \mathbf{Z}$

where $\mathbf{J}$ is the $n \times n$ Jacobian matrix defined by $J_{i j}=\partial f_{i}$ / $\partial Z_{j}$. Then, starting from a unit vector $\delta \mathbf{Z}(0)$, the original system given by Eq. 7 is integrated for $\mathbf{Z}$ together with the $\delta \mathbf{Z}$ tangent system given by Eq. 8. The evolution of $\delta \mathbf{Z}$ is such that it tends to align with the most unstable direction (the most rapidly growing one). The choice of the initial vector of the tangent manifold may influence the convergence, but in practice a spin-up phase can be performed in order to find the good direction.

The largest Lyapunov exponent $\lambda_{\max }$ is then defined as:

$\lambda_{\text {max }}=\lim _{|\delta \mathbf{Z}(0)| \rightarrow 0} \lim _{t \rightarrow \infty} \frac{1}{t} \ln \frac{|\delta \mathbf{Z}(t)|}{|\delta \mathbf{Z}(0)|}$

It is of course impossible in practice to go to infinity ${ }^{13}$; so the computation is always truncated to some finite final time, usually of the order of $10^{4-5}$ times the period of the forcing. The convergence can be more rapidly achieved if some transient behaviour is skipped (like illustrated in Fig. 17), i.e. we compute the LCEs only when we are quite sure to be on the attracting trajectory.

But a dynamical system of dimension $n$ has $n$ LCEs and $n$ eigenvectors (Lichtenberg and Lieberman 1983): there exists a whole spectrum of Lyapunov exponents $\lambda_{i}$ $(i=1,2, \ldots, n)$. This spectrum can be computed with a unit vector basis, and with a renormalisation procedure. Although we are mostly interested in $\lambda_{\max }$ in this article-a positive $\lambda_{\text {max }}$ is associated with a desynchronisation-, our subroutine allows to compute all the spectrum of $\lambda_{i}$ 's of any system. For the sake of flexibility, we used a symbolic software, so that the model could be very easily changed, and all functions (like the Jacobian matrix) are automatically derived once the initial system is given.

\footnotetext{
${ }^{13}$ The proof of the existence of such a limit has been given by Oseledec (1968).
} 
One of the standard and popular methods to compute the Lyapunov spectrum of a dynamical system involves a Gram-Schmidt reorthonormalisation (GSR) of the 'tangent vectors' (Shimada and Nagashima 1979; Benettin et al. 1980; Wolf et al. 1985); differential versions of this have also been formulated. Our subroutine includes the GSR procedure, which is required in order to avoid computational overflows, and degeneracy into a single vector. The frequency of reorthonormalisation is not critical; as a rule of thumb, GSR is usually performed on the order of once per characteristic period. Here, the normalisation time step has been chosen optimally, that is to say the largest possible which preserves the accuracy. Since the GSR never affects the direction of the first vector in a system, this vector tends to seek out the direction in tangent space which is most rapidly growing.

Our subroutine has been validated by comparing our LCEs to those of Ramasubramanian and Sriram (2000) for several systems (driven van der Pol, Lorenz '63, etc.); the order of the accuracy achieved is $1 \%$ o for the Lorenz system $^{14}$. One also checked that $\operatorname{div} \mathbf{f}=\operatorname{Tr}(\mathbf{J})=-\sigma-1-$ $\beta=-13.66=\sum_{i=1}^{n} \lambda_{i}$ holds. Note that $\operatorname{div}(\mathbf{f})<0$ for a dissipative system (hence the system has at least one negative exponent).

Coming back to our system of Eqs. $4 \mathrm{a}, 4 \mathrm{~b}$, for which the Jacobian matrix is:

$\mathbf{J}=-\left[\begin{array}{cc}0 & 1 \\ -\alpha & \alpha \Phi^{\prime \prime}(y)\end{array}\right] / \tau=\mathbf{J}(y, \alpha)$

we end up with the following results (Fig. 17): the system Eqs. 4a, 4b subject to the insolation (Eq. 1) is synchronised on a long term, since $\lambda_{\max }=-0.2 \mathrm{kyr}^{-1}<0$.

Note finally that the LCEs are very useful in order to characterise the dynamical behaviour of a system and that many theorems exist on several interesting properties; local bifurcations can also be detected by detecting changes of signs of $\lambda_{i}$. It is also possible to estimate the LCE of a system by analysing time series of a single realisation with limited data, or a system subject to non-negligible stochastic perturbation, where the classical methods may provide incorrect or ambiguous results (Ruelle 1990), hence require specific methods, like McCaffrey et al. (1992) and Liu et al. (2005).

\section{Appendix 3: Technical details about the computation of the basins of attraction}

The practical computation of a basin of attraction is done as follows.

\footnotetext{
${ }^{14}$ For the Lorenz system, one must pay attention to the spurious trivial set of LCEs corresponding to the origin (Shimada and Nagashima 1979; Bryant et al. 1990).
}

Let us for example come back to Fig. 5e, with the three attracting trajectories $A T_{i}(x, y, t)$ due to the insolation forcing.

Now, we wonder which initial condition leads ultimately to which of the three $A T_{i}$. This is the concept of the basin of attraction of a given $A T_{i}$, classically defined by the set of states that leads to a given $A T_{i}$. Let us more precisely define the basin of attraction of a given $A T_{i}$ as the locus of all points in the $(x, y)$ plane which lead to trajectories which ultimately converge on that $A T_{i}$.

We initialise many initial conditions on a fine rectangular grid $(201 \times 121=24321$ points here $)$ covering the phase space. Each initial condition is then integrated forward to see which $A T_{i}$ its trajectory approached. If the trajectory approaches a particular one of the three $A T_{i}$ 's, a dot coloured by the colour identifying the $A T_{i}$ is plotted on the grid. For doing this, we need to define a target time at which we will do the classification, and a criterion for the classification.

The classification algorithm is illustrated in Fig. 18, where a cut has been made at a target time $t=550 \mathrm{kyr}$. The three $A T_{i}$ 's are displayed, together with the location of the trajectories (black circles). To decide if a given trajectory ends up onto a given $A T_{i}$, we choose a maximum distance from the $A T_{i}$ (dotted circle); taken here to be $1 / 4$ of the minimum distance between two $A T_{i}$ 's. Trajectories falling into that dotted circle are classified as ending into $A T_{i}$.

We consider a given trajectory starting from $t_{0}=0 \mathrm{kyr}$, integrate it up to $t=550 \mathrm{kyr}$, and then we examine its position with respect to the $A T_{i}$ at the same time $t=550 \mathrm{kyr}$. If the distance to a given $A T_{k}$ is 'sufficiently small' (see the circles around the $A T_{i}$ ), then this initial condition is coloured in the kth colour, associated with the kth attracting trajectories $A T_{i}$. Repeating this process for each initial condition on a fine grid of the whole phase space gives the geometry of the basins of attraction. Of course, there are as many basins of attraction as attracting trajectories.

As we are in the case of a non-autonomous system, we then have to repeat this procedure for several starting times $t_{0}$ (the position of the $A T_{i}$ 's are constantly evolving, hence the geometry of the basins are also varying with time). This has been done to produce the evolving geometry of the basins of attraction in Figs. 11 (41-kyr periodic forcing) and 12 (quasiperiodic insolation forcing).

Note that if $t$ is too close from $t_{0}$, transient behaviours predominate, and not enough time has elapsed in order for the trajectory to be attracted by a given $A T_{i}$, hence the basins of attraction cannot be defined in that case.

The glacial/interglacial cycles do exist since about 3 million years, but only 8 limit cycles of $100 \mathrm{kyr}$ period have been performed, since the Mid-Pleistocene Transition. This 
should however be sufficient to converge onto the attracting trajectories, because the climatic trajectories are rapidly attracted on the limit cycle. So, if the ice age model Eqs. 4a, $4 \mathrm{~b}$ is a realistic one, it would be reasonable to state that the transient of climate dynamics has gone, and that we are currently probably somewhere on an attracting trajectory, if any.

\section{References}

Abarbanel HDI, Brown R, Kennel MB (1991) Variation of Lyapunov exponents on a strange attractor. J Nonlinear Sci 1(2):175-199

Abarbanel HDI, Rulkov NF, Sushchik MM (1996) Generalized synchronization of chaos: the auxiliary system approach. Phys Rev E 53(5):4528-4535

Arnold V (1983) Geometrical methods in the theory of ordinary differential equations. Springer, New York (1988 second edition. English translation of the original russian publication: "Dopolnitel'nye Glavy Teorii Obyknovennykh Differentsial'nykh Uravnenî̂" (Additional Chapters to the Theory of Ordinary Differential Equations, Moscow: Nauka, 1978))

Ashkenazy Y (2006) The role of phase locking in a simple model for glacial dynamics. Clim Dyn 27(4):421-431

Balanov A, Janson N, Postnov D, Sosnovtseva O (2009) Synchronization: from simple to complex. Springer, Berlin

Barnes B, Grimshaw R (1997) Analytical and numerical studies of the bonhoeffer van der Pol system. ANZIAM J 38(04):427-453

Belogortsev AB (1992) Quasiperiodic resonance and bifurcations of tori in the weakly nonlinear duffing oscillator. Physica D 59(4):417-429

Benettin G, Galgani L, Giorgilli A, Strelcyn J-M (1980) Lyapunov characteristic exponents for smooth dynamical systems and for hamiltonian systems; a method for computing all of them. part 2: Numerical application. Meccanica 15(1):21-30

Benoît E, Callot J, Diener F, Diener M (1981) Chasse au canard. Collectanea Mathematica 31-32(1-3):37-119

Benzi R, Parisi G, Sutera A, Vulpiani A (1982) Stochastic resonance in climatic change. Tellus 34(1):10-16

Berger AL (1978) Long-term variations of daily insolation and quaternary climatic changes. J Atmos Sci 35:2362-2367

Braun H, Ditlevsen P, Kurths J (2009) New measures of multimodality for the detection of a ghost stochastic resonance. Chaos 19(4):043132

Broecker WS, van Donk J (1970) Insolation changes, ice volumes, and the $\mathrm{O} 18$ record in deep-sea cores. Rev Geophys 8(1):169-198

Broer HW, Simó C (1998) Hill's equation with quasi-periodic forcing: resonance tongues, instability pockets and global phenomena. Bol Soc Brasil Mat (N.S.) 29:253-293

Brown R, Kocarev L (2000) A unifying definition of synchronization for dynamical systems. Chaos 10(2):344-349

Bryant P, Brown R, Abarbanel HDI (1990) Lyapunov exponents from observed time series. Phys Rev Lett 65(13):1523-1526

Chen J-H, Chen W-C (2008) Chaotic dynamics of the fractionally damped van der Pol equation. Chaos Solit Fract 35(1):188-198

Crucifix M (2012) Oscillators and relaxation phenomena in Pleistocene climate theory. Philos Trans R Soc A 370:1140-1165

D'Acunto M (2006) Determination of limit cycles for a modified van der pol oscillator. Mech Res Commun 33(1):93-98

DegliEsposti Boschi C, Ortega GJ, Louis E (2002) Discriminating dynamical from additive noise in the van der pol oscillator. Physica D 171(1-2):8-18
Dijkstra HA, Weijer W, Neelin JD (2003) Imperfections of the threedimensional thermohaline circulation: hysteresis and uniquestate regimes. J Phys Oceanogr 33:2796-2814

Doedel E, Champneys A, Dercole F, Fairgrieve T, Kuznetsov Y, Oldeman B, Paffenroth R, Sandstede B, Wang X, Zhang C (2009) Auto: software for continuation and bifurcation problems in ordinary differential equations. Technical report, Montreal

Donges JF, Zou Y, Marwan N, Kurths J (2009) The backbone of the climate network. EPL 87(4):48007

Feudel U, Kurths J, Pikovsky AS (1995) Strange non-chaotic attractor in a quasiperiodically forced circle map. Physica D 88(3-4): 176-186

Ganopolski A, Rahmstorf S (2002) Abrupt glacial climate changes due to stochastic resonance. Phys Rev Lett 88(3):038501

Gildor H, Tziperman E (2000) Sea ice as the glacial cycles climate switch: role of seasonal and orbital forcing. Paleoceanography 15:605-615

Ginoux J-M, Rossetto B (2006) Differential geometry and mechanics: Applications to chaotic dynamical systems. Int J Bifurcat Chaos 16(4):887-910

Glass L, Mackey M (1988) From clocks to chaos: the rhytms of life. Princeton University Press, Princeton

Glass L, Sun J (1994) Periodic forcing of a limit-cycle oscillator: fixed points, Arnold tongues, and the global organization of bifurcations. Phys Rev E 50:5077-5084

Glendinning P, Wiersig J (1999) Fine structure of mode-locked regions of the quasi-periodically forced circle map. Phys Lett A 257(1-2):65-69

Grasman J, Verhulst F, Shih S (2005) The Lyapunov exponents of the Van der Pol oscillator. Math Methods Appl Sci 28:1131-1139

Grebogi C, Ott E, Pelikan S, Yorke JA (1984) Strange attractors that are not chaotic. Physica D 13(1-2):261-268

Guckenheimer J, Haiduc R (2005) Canards at folded node. Mosc Math J 5:91-103

Guckenheimer J, Holmes P (1983) Nonlinear oscillations, dynamical systems, and bifurcations of vector fields. Springer, New York

Guckenheimer J, Hoffman K, Weckesser W (2000) Numerical computation of canards. Int J Bifurcat Chaos 10(12):2269-2687

Hays JD, Imbrie J, Shackleton NJ (1976) Variations in the earth's orbit: pacemaker of ice ages. Science 194:1121-1132

Hilborn R (2000) Chaos and nonlinear dynamics: an introduction for scientists and engineers. Oxford University Press, Oxford

Huybers P (2007) Glacial variability over the last two millions years: an extended depth-derived age model, continuous obliquity pacing, and the Pleistocene progression. Quat Sci Rev 26:37-55

Hyde WT, Peltier WR (1985) Sensitivity experiments with a model of the ice age cycle: the response to harmonic forcing. J Atmos Sci 42(20):2170-2188

Hyde WT, Peltier WR (1987) Sensitivity experiments with a model of the ice age cycle: the response to Milankovitch forcing. J Atmos Sci 44(10):1351-1374

Imbrie J, Imbrie JZ (1980) Modelling the climatic response to orbital variations. Science 207:943-953

Kantz H, Schreiber T (2004) Nonlinear time series analysis, 2nd edn. Cambridge University Press, Cambridge

Kloeden PE (2000) A Lyapunov function for pullback attractors of nonautonomous differential equations. Electronic J Diff Eqns Conf 05:91-102

Kosmidis EK, Pakdaman K (2003) An analysis of the reliability phenomenon in the fitzhugh-nagumo model. J Comput Neurosci 14(1):5-22

Langa JA, Robinson JC, Suárez A (2002) Stability, instability, and bifurcation phenomena in non-autonomous differential equations. Nonlinearity 15(3):1-17

Laskar J, Robutel P, Joutel F, Boudin F, Gastineau M, Correia ACM, Levrard B (2004) A long-term numerical solution for the 
insolation quantities of the earth. Astronom Astroph 428:261-285

Le Treut H, Ghil M (1983) Orbital forcing, climatic interactions and glaciation cycles. J Geophys Res 88(C9):5167-5190

Lichtenberg AJ, Lieberman MA (1983) Regular and stochastic motion. Springer, New York

Lisiecki LE, Raymo ME (2005) A pliocene-pleistocene stack of 57 globally distributed benthic $\delta^{18} O$ records. Paleoceanography 20:PA1003

Lisiecki LE, Raymo ME (2007) Plio-pleistocene climate evolution: trends and transitions in glacial cycle dynamics. Quat Sci Rev 26(1-2):56-69

Liu H-F, Dai Z-H, Li W-F, Gong X, Yu Z-H (2005) Noise robust estimates of the largest Lyapunov exponent. Phys Lett A 341(1-4):119-127

Luethi D, Le Floch M, Bereiter B, Blunier T, Barnola J-M, Siegenthaler U, Raynaud D, Jouzel J, Fischer H, Kawamura K, Stocker TF (2008) High-resolution carbon dioxide concentration record 650,000-800,000 years before present. Nature 453(7193): 379-382

Marwan N, Donges JF, Zou Y, Donner RV, Kurths J (2009) Complex network approach for recurrence analysis of time series. Phys Lett A 373(46):4246-4254

McCaffrey DF, Ellner S, Gallant AR, Nychka DW (1992) Estimating the Lyapunov exponent of a chaotic system with nonparametric regression. J Am Stat Assoc 87(419):682-695

Mettin R, Parlitz U, Lauterborn W (1993) Bifurcation structure of the driven van der Pol oscillator. Int J Bifurcat Chaos 3(6):1529_ 1555

Milankovitch M (1941) Kanon der Erdbestrahlung und seine Anwendung auf das Eiszeitenproblem. Königlich Serbische Akademie, Belgrade

Oseledec V (1968) A multiplicative ergodic theorem: Ljapunov characteristic numbers for dynamical systems. Trans Moscow Math Soc 19:197-231

Osinga H, Wiersig J, Glendinning P, Feudel U (2000) Multistability and nonsmooth bifurcations in the quasiperiodically forced circle map. ArXiv Nonlinear Sci e-prints: http://arxiv.org/abs/nlin/ $0005032 \mathrm{v} 1$

Ott E (2002) Chaos in dynamical systems. Cambridge University Press, Cambridge

Paillard D (1998) The timing of pleistocene glaciations from a simple multiple-state climate model. Nature 391:378-381

Paillard D (2001) Glacial cycles: toward a new paradigm. Rev Geophys 39(3):325-346

Paillard D, Parrenin F (2004) The Antarctic ice sheet and the triggering of deglaciations. Earth Planet Sci Lett 227:263-271

Parlitz U, Lauterborn W (1987) Period-doubling cascades and devil's staircases of the driven van der pol oscillator. Phys Rev A 36(3):1428-1434

Pikovsky A, Rosenblum M, Kurths J (2001) Synchronization: a universal concept in nonlinear sciences. Cambridge University Press, New York

Rahmstorf S, Crucifix M, Ganopolski A, Goosse H, Kamenkovich I, Knutti R, Lohmann G, Marsh R, Mysak LA, Wang Z, Weaver AJ (2005) Thermohaline circulation hysteresis: a model intercomparison. Geophys Res Lett 32:L23605

Ramasubramanian K, Sriram MS (2000) A comparative study of computation of Lyapunov spectra with different algorithms. Physica D 139(1-2):72-86

Rial JA, Saha R (2011) Modeling abrupt climate change as the interaction between sea ice extent and mean ocean temperature under orbital insolation forcing. In: Rashid H, Polyak L, Mosley-
Thompson E (eds) AGU geophysics monograph 193, understanding the causes, mechanisms and extent of abrupt climate change, pp 57-74

Rial JA, Yang M (2007) Is the frequency of abrupt climate change modulated by the orbital insolation? In: Hamming S (eds) AGU monograph 173, ocean circulation, mechanisms and impacts, pp 167-174

Rosenstein MT, Collins JJ, Luca CJD (1993) A practical method for calculating largest Lyapunov exponents from small datasets. Physica D 65:117-134

Ruelle D (1990) Deterministic chaos: the science and the fiction. Proc R Soc A Lond 427:241-248

Ruihong L, Wei X, Shuang L (2008) Chaos control and synchronization of the $\phi^{6}$-van der pol system driven by external and parametric excitations. Nonlinear Dyn 53(3):261-271

Rulkov NF, Sushchik MM, Tsimring LS, Abarbanel HDI (1995) Generalized synchronization of chaos in directionally coupled chaotic systems. Phys Rev E 51(2):980-994

Saltzman B (2002) Dynamical paleoclimatology: generalized theory of global climate change (international geophysics). Academic Press, London

Saltzman B, Maasch KA (1990) A first-order global model of late Cenozoic climate. Trans R Soc Edinburgh Earth Sci 81:315-325

Saltzman B, Maasch KA (1991) A first-order global model of late Cenozoic climate. II further analysis based on a simplification of the $\mathrm{CO}_{2}$ dynamics. Clim Dyn 5:201-210

Saltzman B, Hansen AR, Maasch KA (1984) The late Quaternary glaciations as the response of a 3-component feedback-system to earth-orbital forcing. J Atmos Sci 41(23):3380-3389

Savi MA (2005) Chaos and order in biomedical rhythms. J Braz Soc Mech Sci Eng 27(2):157-169

Shimada I, Nagashima T (1979) A numerical approach to ergodic problem of dissipative dynamical systems. Prog Theor Phys 61(6): 1605-1616

Strogatz SH (1994) Nonlinear dynamics and chaos: with applications to physics, biology, chemistry, and engineering (studies in nonlinearity). Studies in nonlinearity, 1st edn. Perseus Books Group, Cambridge

Svensson C-M, Coombes S (2009) Mode locking in a spatially extended neuron model: active soma and compartmental tree. Int J Bifurcat Chaos 19(8):2597-2607

Tziperman E, Gildor H (2003) On the mid-Pleistocene transtion to $100-\mathrm{kyr}$ glacial cycles and the asymmetry between glaciation and deglaciation times. Paleoceanography 18(1):1001

Tziperman E, Raymo ME, Huybers P, Wunsch C (2006) Consequences of pacing the Pleistocene $100 \mathrm{kyr}$ ice ages by nonlinear phase locking to Milankovitch forcing. Paleoceanography 21:PA4206

van der Pol B (1926) On relaxation oscillations. Phil Mag 2(11):978992

Wieczorek S (2009) Stochastic bifurcation in noise-driven lasers and Hopf oscillators. Phys Rev E 79(3):036209

Wieczorek SM (2011) Noise synchronisation and stochastic bifurcations in lasers. http://arxiv.org/abs/1104.4052

Wiggins S (2003) Introduction to applied nonlinear dynamical systems and chaos. Texts in applied mathematics, 2nd edn. Springer, Berlin

Wolf A, Swift JB, Swinney HL, Vastano JA (1985) Determining Lyapunov exponents from a time series. Physica D 16(3):285317

Wu L, Zhu S, Li J (2006) Synchronization on fast and slow dynamics in drive-response systems. Physica D 223(2):208-213 